100

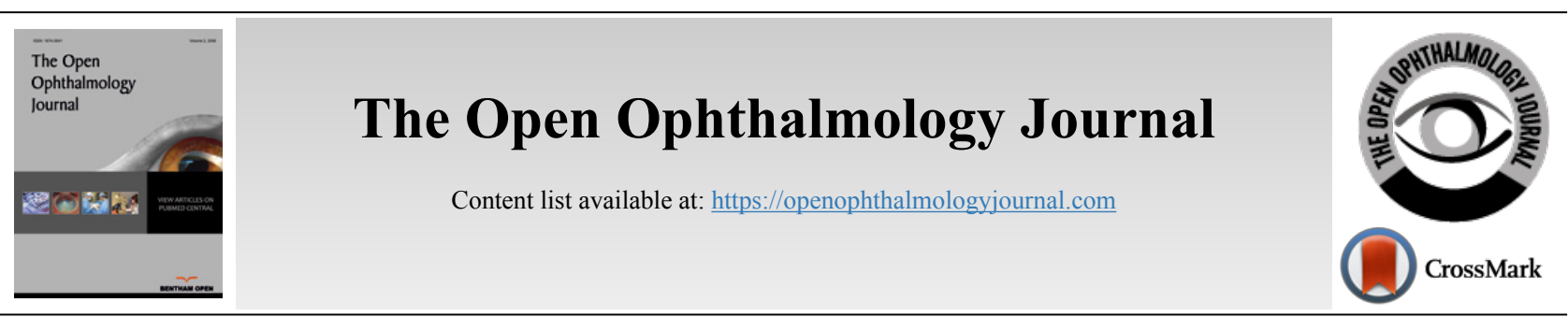

REVIEW ARTICLE

\title{
The Spectrum of Microbial Keratitis: An Updated Review
}

\author{
Christopher Bartimote ${ }^{1,2,}$, John Foster $^{3}$ and Stephanie Watson ${ }^{1,2}$ \\ ${ }^{I}$ Department of Ophthalmology, Sydney Eye Hospital, Sydney, New South Wales, Australia \\ ${ }^{2}$ The University of Sydney, Save Sight Institute, Discipline of Ophthalmology, Sydney Medical School, Sydney, New South Wales, Australia \\ ${ }^{3}$ Department of Chemistry, University of Alabama in Huntsville, Huntsville, Alabama, USA
}

\begin{abstract}
:
Background:

In microbial keratitis, infection of the cornea can threaten vision through permanent corneal scarring and even perforation resulting in the loss of the eye. A literature review was conducted by Karsten, Watson and Foster (2012) to determine the spectrum of microbial keratitis. Since this publication, there have been over 2600 articles published investigating the causative pathogens of microbial keratitis.
\end{abstract}

Objective:

To determine the current spectrum of possible pathogens implicated in microbial keratitis relative to the 2012 study.

Methods:

An exhaustive literature review was conducted of all the peer-reviewed articles reporting on microbial pathogens implicated in keratitis. Databases including MEDLINE, EMBASE, Scopus and Web of Science were searched utilising their entire year limits (1950-2019).

Results:

Six-hundred and eighty-eight species representing 271 genera from 145 families were implicated in microbial keratitis. Fungal pathogens, though less frequent than bacteria, demonstrated the greatest diversity with 393 species from 169 genera that were found to cause microbial keratitis. There were 254 species of bacteria from 82 genera, 27 species of amoeba from 11 genera, and 14 species of virus from 9 genera, which were also identified as pathogens of microbial keratitis.

\section{Conclusion:}

The spectrum of pathogens implicated in microbial keratitis is extremely diverse. Bacteria were most commonly encountered and in comparison, to the review published in 2012, further 456 pathogens have been identified as causative pathogens of microbial keratitis. Therefore, the current review provides an important update on the potential spectrum of microbes, to assist clinicians in the diagnosis and treatment of microbial keratitis.

Keywords: Microbial keratitis, Staphylococcus, Bacterial keratitis, Acanthamoeba, Mycotic keratitis, Pseudomonas, Herpetic keratitis.

Article History

Received: August 09, 2019

Revised: October 22, 2019

Accepted: October 28, 2019

\section{INTRODUCTION}

Microbial keratitis is a common infectious disease of the cornea that, if untreated, can have severe consequences [1 - 3]. Consequently, keratitis is considered an ophthalmic emergency requiring immediate and appropriate anti-microbial treatment to prevent permanent vision loss [4]. Current practice is to combat infection through the application of empiric broadspectrum anti-microbial therapy, which is instituted immediate-

* Address correspondence to this author at the Department of Ophthalmology, Sydney Eye Hospital, Sydney, New South Wales, Australia; Tel: 0481467283; E-mail: chrisbartimote90@gmail.com ly after corneal scrapings to combat the infection while cultures are processed $[3,5,6]$. Complications, however, may arise due to misidentification of the microbial cause and subsequent inadequate and/or inappropriate treatment $[7,8]$. With definitive identification and treatment, the significant morbidity associated with severe microbial keratitis may be avoided.

In 2012, Karsten, Watson \& Foster conducted a literature review investigating the spectrum of pathogens implicated in microbial keratitis. According to their review of the literature, 232 species from 142 genera representing 80 families were implicated in microbial keratitis, with bacterial keratitis, the 
most common. Since this publication, however, there have been over 2700 articles published investigating the identity and aetiology of causative pathogens of microbial keratitis. Therefore, an updated review is required to provide clinicians with current data on causative pathogens and management of microbial keratitis.

The aim of this study was to determine the current spectrum of possible pathogens implicated in microbial keratitis relative to the 2012 study, as well as the aetiological factors and treatment of this eye-threatening disease.

\section{MATERIALS AND METHODS}

A review of peer-reviewed articles, case reports and conference submissions reporting microbial keratitis pathogens was conducted. The search strategy; 'keratitis', 'microbial keratitis', 'bacterial keratitis', 'viral keratitis', 'fungal keratitis', 'amoebic keratitis' OR 'parasitic keratitis' was used in MEDLINE, EMBASE, Scopus and Web of Science databases within the year limits of each of the databases. Searches were restricted to the English language. The most recent search was conducted in August 2019.

The search strategy generated over 12600 articles, case reports and conference submissions to be reviewed. Publications were reviewed and information reporting the identification of organisms that were implicated in microbial keratitis were abstracted on the basis of (a) organism identity, (b) aetiology and (c) treatment. Pathogens were included in the review only if the method of the article outlined that the pathogen was retrieved from a corneal biopsy or scraping and cultured appropriately. The review objective was restricted to species diversity and did not aim to compile any incidence data.

\section{RESULTS AND DISCUSSION}

In microbial keratitis, a range of microorganisms, including fungi, bacteria, protozoa, and viruses, have been identified as infectious agents. A comprehensive review of the current literature identified 688 species representing 271 genera from 145 families reported to cause microbial keratitis. According to the literature, fungal keratitis, although less common than bacterial keratitis, demonstrated the greatest diversity of pathogens with 393 species from 169 genera being implicated in causing keratitis. Moreover, 254 species of bacteria from 82 genera, 27 species of amoeba from 11 genera, and 14 species of virus from 9 genera were found to be pathogens of microbial keratitis (Fig 1).

In total, 49 bacterial families were identified; 22 were Gram-positive and 27 Gram-negative with Proteobacteria phylum being the most common (Table 1). Eighty-two fungal families were identified, the most common were filamentous fungi (76 families, Table 2). In viral keratitis, 5 families were reported with the majority of the species belonging to the Herpesviridae family. In amoebic keratitis, 8 families were identified to cause microbial keratitis, the most common was Acanthamoeba species.

The presentation of keratitis is often similar between the pathogens, with photophobia, pain, lacrimation and foreign body sensation all common presenting symptoms [9]. The clinical appearance of the cornea, however, may differ depending on the cause of the keratitis $[7,10]$.

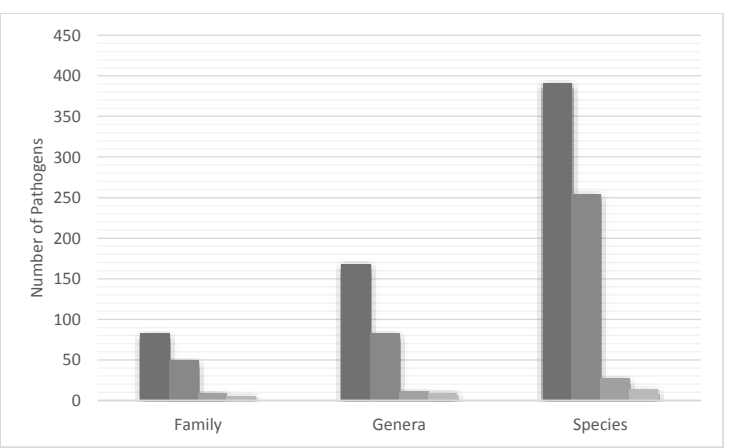

Fig. (1). Distribution of microbial pathogens implicated in keratitis Fungal, Bacterial, Amoeba, Viral.

Table 1. Number of identified families in bacterial.

\begin{tabular}{|c|c|c|}
\hline & Phylum & $\begin{array}{c}\text { Number of Identified } \\
\text { Families }\end{array}$ \\
\hline \multirow{2}{*}{ Gram Positive } & Actinobacteria & 10 \\
\cline { 2 - 3 } & Firmicutes & 12 \\
\hline \multirow{2}{*}{ Gram Negative } & Bacteriodetes & 5 \\
\cline { 2 - 3 } & Fusobacteria & 1 \\
\cline { 2 - 3 } & Proteobacteria & 20 \\
\cline { 2 - 3 } & Spirochaetae & 1 \\
\hline
\end{tabular}

Table 2. Number of identified families in fungal keratitis.

\begin{tabular}{|c|c|c|}
\hline & Phylum & Number of Identified Families \\
\hline \multirow{3}{*}{ Mycota } & Ascomycota & 59 \\
\cline { 2 - 3 } & Basiiomycota & 16 \\
\cline { 2 - 3 } & Zygomycota & 5 \\
\hline Slime Mold & Myoxmycota & 1 \\
\hline Straminipila & Oomycota & 2 \\
\hline
\end{tabular}

\subsection{Bacterial Keratitis}

Bacterial pathogens described in the literature were responsible for a greater proportion of keratitis than mycotic pathogens throughout various populations [11 - 13]. Bacterial keratitis often occurs in patients with inherent ocular risk factors, such as ocular trauma, contact lens use, or corneal disease [4]. Moreover, with the subsequent increased use of prescription and aesthetic contact lenses, there has been an increased prevalence of bacterial keratitis [14].

In this review of the literature, the Gram-positive bacteria identified were almost equally spread between the phylum Actinobacteria and Firmicutes. However, consistent with Karsten, Watson \& Foster (2012), the Gram-negative bacteria identified were largely from the phylum Proteobacteria (Table 1). The most diverse species of bacteria were Nocardia, Staphylococcus, and Streptococcus with 24, 19 and 14 species, respectively (Appendix 1). Gram-positive bacteria such as coagulase-negative Staphylococcal (CoNS) species were more common [4] than Gram-negative bacteria as a cause of bacterial keratitis [3, 15]. Although, in contact lens wearers Pseudomonas aeruginosa was the most common cause [4, 14] (Table 3). 
Table 3. Families implicated in bacterial keratitis, new families in bold.

\begin{tabular}{|c|c|c|c|}
\hline Family & References & Family & References \\
\hline Acetobacteraceae & {$[25]$} & Listeriaceae & {$[26]$} \\
\hline Actinomycetaceae & {$[27,28]$} & Microbacteriaceae & {$[29,30]$} \\
\hline Aerococcaceae & {$[31-33]$} & Micrococcaceae & {$[10,30,34-40]$} \\
\hline Aeromonadaceae & {$[15,41,42]$} & Moraxellaceae & {$[30,43-50]$} \\
\hline Alcaligenaceae & {$[12,33,42,51,52]$} & Mycobacteriaceae & {$[47,53-59]$} \\
\hline Bacillaceae & {$[60-64]$} & Neisseriaceae & {$[13,51,65-67]$} \\
\hline Bacteroidaceae & {$[68]$} & Nocardiaceae & {$[44,69-86]$} \\
\hline Bartonellaceae & {$[87]$} & Pasteurellaceae & {$[41,44,46,88$ - 91$]$} \\
\hline Brevibacteriaceae & {$[12]$} & Peptococcaceae & {$[92]$} \\
\hline Brucellaceae & {$[49,93,94]$} & Porphyromonadaceae & {$[95]$} \\
\hline Burkholderiaceae & {$[47,96,97]$} & Propionibacteriaceae & {$[1,68,98,99]$} \\
\hline Cardiobacteriaceae & {$[34]$} & Prevotellaceae & {$[68,100]$} \\
\hline Carnobacteriaceae & [101] & Pseudomonadaceae & {$[30,33,38,52,102-107]$} \\
\hline Caulobacteraceae & {$[108]$} & Rhizobiaceae & {$[109]$} \\
\hline Clostridiaceae & {$[60,68,110-112]$} & Rhodobacteraceae & [113] \\
\hline Comamonadaceae & {$[55,98]$} & Rickettsiaceae & {$[114]$} \\
\hline Corynebacteriaceae & {$[15,44,62,105,115-121]$} & Sphingobacteriaceae & {$[30]$} \\
\hline Enterobacteriaceae & $\begin{array}{c}{[33,34,43,52,61,65,102,104,105,117,122} \\
-136]\end{array}$ & Spirochaetaceae & {$[136,137]$} \\
\hline Enterococcaceae & {$[33,39,138]$} & Staphylococcaceae & {$[10,15,23,33,34,36,39,43,46,52,60,138$ - 142] } \\
\hline Eubacteriaceae & [143] & Streptococcaceae & {$[33,41,46,47,49,60,64,65,117,126,144-146]$} \\
\hline Flavobacteriaceae & {$[34,52,147-155]$} & Streptomycetaceae & {$[131,156]$} \\
\hline Fusobacteriaceae & {$[157]$} & Tsukamurellaceae & {$[158,159]$} \\
\hline Intrasporangiaceae & [160] & Vibrionaceae & {$[43,161]$} \\
\hline Lactobacillaceae & [162] & Xanthomonadaceae & {$[46,98,163]$} \\
\hline Leuconostocaceae & {$[10]$} & Yersiniaceae & {$[43,164]$} \\
\hline
\end{tabular}

Table 4. Families implicated in fungal keratitis, new families in bold.

\begin{tabular}{|c|c|c|c|}
\hline Family & References & Family & References \\
\hline Ajellomycetaceae & {$[171,180,181]$} & Hypocreales Incertae sedis & {$[102,146,175]$} \\
\hline Amphisphaeriaceae & {$[182]$} & Hyponectriaceae & [12] \\
\hline Arthrodermataceae & {$[171,181,183-188]$} & Glomerellaceae & {$[41,78,169,189-195]$} \\
\hline Ascodesmiaceae & {$[186,196]$} & Gymnoascaceae & {$[197]$} \\
\hline Basidiobolaceae & {$[187]$} & Lagenidiaceae & [198] \\
\hline Bionectriaceae & {$[186]$} & Lasiosphaeriaceae & {$[169,173,186,193,199,200]$} \\
\hline Botryosphaeriaceae & {$[11,169,175,176,188,191,196,201-209]$} & Lichtheimiaceae & {$[193,204]$} \\
\hline Cephalothecaceae & {$[202,210]$} & Lophiostomataceae & {$[211]$} \\
\hline Ceratobsidiaceae & {$[169,175]$} & Malasseziaceae & {$[175,212,213]$} \\
\hline Chaetomiaceae & {$[12,47,168,169,191,204,205,214]$} & Massarinaceae & {$[10,90]$} \\
\hline Chaetosphaerellaceae & {$[175]$} & Metacapnodiaceae & {$[186]$} \\
\hline Chaetosphaeriaceae & {$[186,206]$} & Microascaceae & $\begin{array}{c}{[28,29,47,174,175,186,189,191,196,212,} \\
215-221]\end{array}$ \\
\hline Clavicipitaceae & {$[222-224]$} & Montagnulaceae & {$[225]$} \\
\hline Coniochaetaceae & {$[28,175,210]$} & Mucoraceae & $\begin{array}{c}{[10,28,29,173,175,186,191,193,219,226-} \\
228]\end{array}$ \\
\hline Cordycipitaceae & {$[47,175,191,229,230]$} & Mycosphaerellaceae & {$[186,227,231,232]$} \\
\hline Corticiaceae & {$[28,233]$} & Nectriaceae & $\begin{array}{c}{[8,11,28,112,127,146,172,173,175,186,} \\
191,193,199,204,234-250]\end{array}$ \\
\hline Corynesporascaceae & {$[251]$} & Niessliaceae & {$[186]$} \\
\hline Cryptococcaceae & {$[212,222,252,253]$} & Onygenaceae & {$[29,47,171,175]$} \\
\hline Cunninghamellaceae & {$[186,254]$} & Ophiocordycipitaceae & {$[255]$} \\
\hline
\end{tabular}


(Table 4) contd....

\begin{tabular}{|c|c|c|c|}
\hline Family & References & Family & References \\
\hline Cystofilobasidiaceae & [222] & Ophiostomataceae & {$[228,256]$} \\
\hline Davidiellaceae & {$[115,204,247,257]$} & Orbiliaceae & {$[258]$} \\
\hline Debaryomycetaceae & [259] & Phaeosphaeriaceae & [260] \\
\hline Dermateaceae & [261] & Phycomycetaceae & {$[28]$} \\
\hline Diaporthaceae & {$[262,263]$} & Plectosphaerellaceae & {$[175,264,265]$} \\
\hline Didymellaceae & {$[12,92,175,191,266]$} & Pleosporaceae & $\begin{array}{c}{[34,111,115,169,171,175,190,191,193,199,} \\
204,207,212,220,240,249,267-280]\end{array}$ \\
\hline Dipodascaceae & {$[175,210,281,282]$} & Pleurotheciaceae & [79] \\
\hline Dothioraceae & {$[12,189,207,283]$} & Polyporaceae & {$[80]$} \\
\hline Eremomycetaceae & {$[175,284]$} & Pythiaceae & {$[47,81]$} \\
\hline Gjaerumiaceae & {$[285]$} & Saccharomycetaceae & $\begin{array}{c}{[9,18,48,53,61,82-86,105,125,163-170} \\
175,191,212,222,236,259,286]\end{array}$ \\
\hline Helotailes incertae sedis & {$[187,287]$} & Schizophyllaceae & [288] \\
\hline Herpotrichiellaceae & $\begin{array}{c}{[47,115,146,154,173,175,191,196,206,} \\
240,283,289-295]\end{array}$ & Schizoporaceae & [201] \\
\hline Hypocreaceae & $\begin{array}{c}{[28,127,146,166,175,186,191,193,222,} \\
240,247,270,283,296]\end{array}$ & Sclerotiniaceae & {$[28]$} \\
\hline
\end{tabular}

Bacterial keratitis often presents within an epithelial defect with surrounding corneal infiltrate and stromal oedema [7]. Inflammation within the anterior chamber associated with the keratitis may include a cellular reaction, flare and/or a hypopyon. Progression of bacterial keratitis and outcome tends to relate to the severity of the presentation as well as the potential risk factors for severe disease such as systemic disease and previous ocular history $[4,16]$

The recommendations for empiric therapy for the treatment of bacterial keratitis by the Australian Therapeutic Guidelines are ciprofloxacin $0.3 \%$ or ofloxacin $0.3 \%$ or fortified cefalotin $5 \%$ plus gentamicin $0.9 \%$ [17]. Indeed, a Cochrane review found equal efficacy of such available topical antibiotics for bacterial keratitis. However, an increase in the relative risk of minor adverse events, including discomfort or chemical conjunctivitis was found with aminoglycosides and cephalosporins compared with fluoroquinolones, although there was no difference in serious complications [18 - 22]. Yet, the development of bacterial resistance to antibiotics may lead to the ineffective treatment of bacterial keratitis [23, 24]. Since our prior review (2012) the sole use of fluoroquinolones as primary treatment for bacterial keratitis has increased alongside elevation in fluoroquinolone resistance [24]. Moreover, CoNS and Staphylococcus aureus have shown resistance to cephalotin and fluoroquinolones with the degree of resistance differing around the world [3]. Watson et al. (2018) demonstrated at the Sydney Eye Hospital that CoNS resistance was $9 \%$ to ciprofloxacin, cefatolin and gentamicin. Whereas $\mathrm{Ni}$ et al. (2015) found that resistance at the Wills Eye Hospital in Philadelphia was $32 \%$ to fluoroquinolones. Therefore, the inappropriate use of antibiotics may lead to antimicrobial resistance, prolonged recovery and poor outcomes in bacterial keratitis [3, 24]

\subsection{Fungal Keratitis}

The aetiology of microbial keratitis was found to differ depending on the geographical location of the patient and the infective pathogen [11]. Fungal keratitis is comparatively uncommon in temperate climates, though in tropical areas it can constitute a significant proportion of microbial keratitis [2, 13, 165]. The tropical climate, combined with greater agricultural and vegetation exposure in lower socioeconomic countries causes an increased risk of contracting fungal keratitis in populations $[166,167]$. Moreover, similar to Karsten, Watson \& Foster (2012), the fungal pathogens published in the literature were more often cultured from patients in these areas where they were more commonly exposed to contaminated soils and decaying vegetation while suffering ocular trauma [36, 168 - 170].

Fungi within the classification Mycota were the most common to cause keratitis; of the 83 families identified, only 3 did not belong to this classification (Table 2). Filamentous fungi, consistent with Karsten, Watson \& Foster (2012), were found to be the most common cause of mycotic keratitis with Fusarium and Aspergillus species being the most prevalent $[165,171-173]$ and diverse genera with 38 and 25 species identified, respectively (Appendix 2).

Mycotic keratitis caused by filamentous fungi can involve any area of the cornea and often exhibits grey or yellow-white stromal infiltrate with indistinct margins [7, 174]. Progressive infiltration with multiple granular satellite stromal infiltrates is common and an immune-mediated ring infiltrate around the ulcer is often seen [7]. Yeasts may have a similar clinical presentation to filamentous fungi, however, yeasts tend to cause keratitis during immunosuppression and with systemic diseases such as diabetes, Human Immunodeficiency Virus (HIV), and corticosteroid therapy [174, 175].

The treatment of fungal keratitis consists of topical and systemic anti-fungal therapies $[2,176]$. Natamycin $5 \%$ is often first line, although its effectiveness is limited by its poor penetration into the corneal stroma. Amphotericin B 0.3-0.5\% is an alternative topical therapy but exhibits ocular toxicity [2]. Voriconazole is a proposed third option, however, the 'Mycotic Ulcer Treatment Trial I' found that voriconazole was inferior to natamycin in the treatment of all fungal keratitis, especially Fusarium keratitis [2, 176]. Since our prior review, the Mycotic Ulcer Treatment Trial II demonstrated that there was no therapeutic benefit of adding oral voriconazole to topical 
antifungal therapy [177]. Specifically, oral voriconazole, in addition to topical natamycin and voriconazole did not decrease the rate of corneal perforation nor the need for therapeutic penetrating keratoplasty. The study was halted due to the comparatively higher rates of corneal perforations in the oral voriconazole arm [177]. Filamentous fungi have been most frequently reported in our review, supporting current antifungal treatment recommendations [2, 178, 179] (Table 4).

\subsection{Viral Keratitis}

Viral pathogens are ubiquitous within populations, as evidenced by $90 \%$ of adults being seropositive for Herpes Simplex virus HSV antigens. However, as reported in the literature and Karsten, Watson \& Foster (2012) only 20-30\% of adults develop ocular manifestations from HSV infections [319]. Nonetheless, herpetic eye disease remains a significant cause of blindness worldwide, affecting over 1 million people annually [320].

The clinical features of HSV keratitis depend on the corneal layer affected and can typically include a dendritiform corneal ulcer or significant breakdown of the corneal epithelium with persistent punctate corneal keratopathy and corneal erosions [319]. Following the primary ocular infection, the viral genome may enter the surrounding nerve fibres and travel to the trigeminal nerve ganglion where it will remain in a latent state $[319,321]$. Recurrent HSV infection can then occur in times of immunosuppression as the virus travels from the ganglion to infect the innervated tissue $[319,322]$. Similar to HSV, the Varicella Zoster virus can remain dormant in the nerve ganglion following primary infection. Reactivation of the virus may lead to the development of Herpes Zoster Ophthalmicus [HZO], which presents as painful vesicular rash along the distribution of the innervation of the ophthalmic nerve [322]. Ocular manifestations of HZO can include Herpes Zoster keratitis [323].

From the review of the literature and consistent with Karsten, Watson \& Foster (2012), Herpesviridae were the most common family implicated in viral keratitis. Herpes Simplex Virus Type 1 (HSV-1) was commonly responsible for viral keratitis affecting the skin and mucous membranes in the distribution of the trigeminal nerve [321]. Varicella Zoster virus, Cytomegalovirus, other members of the Herpesviridae family, were also implicated in viral keratitis, along with multiple species of Adenovirus (Table 5).

In the treatment of primary HSV epithelial keratitis acyclovir eye drops were the recommended therapy [17]. However, since Karsten, Watson \& Foster (2012) publication, the Herpetic Eye Disease study II demonstrated that oral acyclovir may be utilised prophylactically to prevent recurrent herpetic keratitis, especially in patients with previous HSV stromal keratitis [319 - 324]. Furthermore, Bhatt et al. [2016], in their Cochrane Systematic review, demonstrated that oral acyclovir was beneficial in the prevention of recurrent herpes simplex keratitis in patients with corneal grafts [321]. Furthermore, the American Academy of Ophthalmology has produced guidelines to aid in the management of HSV Keratitis [325]. Herpes zoster keratitis is treated with oral antivirals, such as acyclovir or valacyclovir, in the first 72 hours to minimise the risk of ocular and other complications [17, 323].

\subsection{Amoebic Keratitis}

Amoebic pathogens are an uncommon cause of keratitis and typically occur in immunocompetent patients associated with ocular trauma and contact lens use [337]. Acanthamoeba, a unicellular protozoan, is a frequent cause of amoebic keratitis [338]. These protozoa exist in polluted soil or contaminated water supplies such as swimming pools, sewage and tap water $[337,339]$. Acanthamoeba occur in both active trophozoite and dormant cyst forms. In favourable environments, the Acanthamoeba trophozoite exhibits high activity, although, in unfavourable environments such as during antibiotic and biocides treatment, Acanthamoeba from cysts with low activity but able to powerfully resist the surrounding environment [337, $338,340]$.

The use of contact lenses, especially if used beyond recommended duration from the manufacturer and during contact with contaminated water, such as swimming, has been associated with a greater risk of Acanthamoeba keratitis [338, 339]. Similarly, ocular trauma with exposure to vegetable matter and contaminated soil heavily contributes to Acanthamoeba keratitis leading to a higher incidence in agricultural populations [167].

Similar to the study by Karsten, Watson \& Foster (2012), Acanthamoeba was found to be the most common amoebic cause of keratitis in the current review [341]. Moreover, it was the most diverse, with 13 species of Acanthamoeba implicated as the cause of keratitis. Acanthamoeba is the most prevalent pathogenic amoeba, however, other families such as Vahlkampfia, Encephalotozoon, Vittaforma and Hartmanella have also been reported to cause amoebic keratitis (Table 6).

Acanthamoeba keratitis is often misdiagnosed as bacterial or mycotic keratitis, leading to inappropriate treatment and poor outcomes [257,339]. Patients often present with pain that ranges from minimal to more commonly disproportionate levels for the clinical features shown [338]. Clinical features are often unilateral, including corneal epitheliopathy, stromal infiltrate, punctate keratopathy, ring infiltrate, perineural infiltrates and pseudo-dendrites [257]. As the disease progresses sterile anterior uveitis with a hypopyon may occur [338].

The prevention of Acanthamoeba keratitis prioritises the strict and appropriate use of contact lenses. Contact lenses must be cleaned and stored in appropriate disinfecting solutions and avoid exposure to potentially contaminated water supplies [338].

The current management of Acanthamoeba keratitis utilises a combination of anti-amoebic therapy, including a biguanide such as polyheramthylen biguanide $0.02 \%$, or chlorhexideine $0.02 \%$ and a diamidine such as propamidine isethionate [339, 342]. It is imperative to institute appropriate therapy quickly to prevent Acanthamoeba penetrating deep into the cornea [338, 342]. However, due to the ability of Acanthamoeba to encyst, Acanthamoeba may remain dormant for extended periods before reactivation. Therefore, therapy combinations utilising diamidines are useful as these are 
effective against trophozoites and cysts [338, 340]. The use of corticosteroid therapy in Acanthamoeba keratitis is controversial, emerging evidence supports its use [343, 344], although there is no clear consensus $[338,345]$. In cases of severe anterior segment inflammation, corticosteroids may be used, however, they must be used judiciously to prevent exacerbation of the infection and suppression of the host immune response [338].

Table 5. Diversity of Viral Keratitis. (-) Species not identified. New pathogens in bold.

\begin{tabular}{|c|c|c|c|}
\hline Family & Genus & Species & References \\
\hline \multirow{4}{*}{ Adenoviridae } & \multirow{4}{*}{ Adenovirus } & - & \multirow{4}{*}[326,327]{} \\
\hline & & Adenovirus-3 & \\
\hline & & Adenovirus-8 & \\
\hline & & Adenovirus-19 & \\
\hline \multirow{5}{*}{ Herpesviridae } & Cytomegalovirus & - & [328] \\
\hline & Lymphocryptovirus & Human herpesvirus 4 & [329] \\
\hline & \multirow{2}{*}{ Simplexvirus } & Herpes Simplex Virus-1 & \multirow{2}{*}[327,330]{} \\
\hline & & Herpes Simplex Virus-2 & \\
\hline & Varicellovirus & Varicella Zoster Virus & [331] \\
\hline \multirow{2}{*}{ Paramyxoviridae } & Morbillivirus & Rubeola virus & [332] \\
\hline & Rubulavirus & Mumps rubulavirus & [333] \\
\hline \multirow{2}{*}{ Picornaviridae } & \multirow{2}{*}{ Enterovirus } & - & \multirow{2}{*}[334]{} \\
\hline & & Enterovirus $B$ & \\
\hline Poxviridae & Orthopoxvirus & Vaccinia Virus & {$[335,336]$} \\
\hline
\end{tabular}

Table 6. Diversity of Amoebic Keratitis. (-) Species not Identified. New Pathogens in Bold.

\begin{tabular}{|c|c|c|c|}
\hline Family & Genus & Species & References \\
\hline \multirow{13}{*}{ Acanthamoebidae } & \multirow{13}{*}{ Acanthamoeba } & Astronyxis & \multirow{13}{*}[341,346-353]{} \\
\hline & & Castellanii & \\
\hline & & Culbertsoni & \\
\hline & & Griffini & \\
\hline & & Hatchetti & \\
\hline & & Lenticulata & \\
\hline & & Lugdunesis & \\
\hline & & Mauritaniensis & \\
\hline & & Palestinensis & \\
\hline & & Polyphaga & \\
\hline & & Quina & \\
\hline & & Rhysodes & \\
\hline & & Triangularis & \\
\hline \multirow{2}{*}{ Demodicidae } & \multirow{2}{*}{ Demodex } & Brevis & \multirow{2}{*}[354]{} \\
\hline & & Folliculorum & \\
\hline \multirow{2}{*}{ Hartmannellidae } & \multirow{2}{*}{ Hartmanella } & - & \multirow{2}{*}[355,356]{} \\
\hline & & Vermiformis & \\
\hline \multirow{3}{*}{ Nosematidae } & Anncaliia & Algerae & {$[357]$} \\
\hline & Nosema & Corneum & {$[358]$} \\
\hline & Vittaforma & Corneae & [359] \\
\hline Onchocercidae & Onchocerca & Volvulus & {$[142]$} \\
\hline Tarsonemidae & Tarsonemid & Heterostigmae & {$[360]$} \\
\hline \multirow{3}{*}{ Unikaryonidae } & \multirow{3}{*}{ Encephalitozoon } & Cuniculi & \multirow{3}{*}[359]{} \\
\hline & & Hellem & \\
\hline & & Intestinalis & \\
\hline \multirow{2}{*}{ Valkampfiidae } & Vahlkampfia & - & {$[361,362]$} \\
\hline & Paravahlkampfia & - & {$[363]$} \\
\hline
\end{tabular}




\section{CONCLUSION}

Microbial keratitis is an ophthalmic emergency as it can lead to irreversible damage to the cornea and loss of vision [1, 3 , 5]. To minimise complications, prompt and effective treatment must be instituted to prevent permanent damage to the cornea. For the successful treatment of microbial keratitis, it is vital to correctly identify the causative organism as it enables the use of the most effective treatments to treat the infection [3].

A comprehensive and exhaustive review of the literature yielded 688 species representing 271 genera from 145 families being implicated in microbial keratitis, in contrast to the 232 species from 142 genera representing 80 families found by Karsten, Watson \& Foster (2012). Similarly, from the current review of the literature, there were 135 microbial species found to cause keratitis published before 2012 that were not identified by Karsten, Watson \& Foster (2012).

Similar to the study by Karsten, Watson \& Foster (2012), the current literature demonstrated that bacterial keratitis was the most common and fungal pathogens were the most diverse, with 391 species identified as causative pathogens of microbial keratitis. Comparatively, there were 254 bacterial species, 27 species of amoeba and 14 viral species found to cause microbial keratitis (Fig 1).

Correspondingly, the geographical variances noted from Karsten, Watson \& Foster (2012) were further reinforced from the literature. It was found that bacterial keratitis is relatively common in developed nations, likely due to the use of contact lenses [14]. Similarly, fungal keratitis is more common in tropical environments and the developing world, most likely due to greater exposure to decaying vegetation and soil [165, 167]. Furthermore, many of the pathogens reported in the literature were often from studies and case reports in patients that had been exposed to poor sanitation, greater agricultural exposure and ocular trauma leading to the development of keratitis [170, 182, 251, 265, 311].

Similarly, the treatment of microbial keratitis has remained APPENDIX similar since Karsten, Watson \& Foster (2012). Bacterial keratitis treatment commonly consists of topical fluoroquinolones, yet there is an increasing risk of antimicrobial resistance [24], necessitating the need for judicious and appropriate use of antimicrobials [3]. Contrastingly, since Karsten, Watson \& Foster (2012), available evidence now suggests that oral antifungals, in addition to topical therapy do not add a therapeutic benefit [177].

This review has demonstrated the great diversity of the pathogens implicated in microbial keratitis. Relative to Karsten, Watson \& Foster (2012), further 443 pathogens were identified as causative organisms of microbial keratitis. Additionally, there has been further investigation of antimicrobial resistance in bacterial keratitis and changes in the management of microbial keratitis. Therefore, the current review provides an important update on the spectrum and management of microbial keratitis, to assist clinicians in its diagnosis and treatment and help reduce the associated morbidity. Advancing the literature into the epidemiology and geographical variance of causative organisms will further aid in the identification and treatment of microbial keratitis.

\section{CONSENT FOR PUBLICATION}

Not applicable.

\section{FUNDING}

None.

\section{CONFLICT OF INTEREST}

The authors declare no conflict of interest, financial or otherwise.

\section{ACKNOWLEDGEMENTS}

Declared none.

Table 1. Diversity of Fungal Keratitis [ $\left[{ }^{1}\right.$ Formerly Wangiella, ${ }^{2}$ Formerly Fusarium fujikuroi, ${ }^{3}$ Previously Fusarium avenacas, ${ }^{4}$ Formerly humicola] [ - ] Species not identified. New pathogens in bold.

\begin{tabular}{|c|c|c|c|}
\hline Family & Genus & Species & References \\
\hline \multicolumn{4}{|c|}{ Fungal Keratitis } \\
\hline \multirow{3}{*}{ Ajellomycetaceae } & Blastomyces & dermatitidis & {$[171]$} \\
\hline & Histoplasma & capsulatum & {$[180]$} \\
\hline & Paracoccidioides & brasiliensis & {$[175]$} \\
\hline Amphisphaeriaceae & Pestalotiopsis & clavispora & {$[182]$} \\
\hline
\end{tabular}




\begin{tabular}{|c|c|c|c|}
\hline Family & Genus & Species & References \\
\hline \multicolumn{4}{|c|}{ Fungal Keratitis } \\
\hline \multirow{9}{*}{ Arthrodermataceae } & Epidermophyton & floccosum & {$[181]$} \\
\hline & \multirow{3}{*}{ Microsporum } & - & \multirow{3}{*}[175,228,364]{} \\
\hline & & canis & \\
\hline & & gypseum & \\
\hline & \multirow{5}{*}{ Trichophyton } & - & \multirow{5}{*}[171,183-185]{} \\
\hline & & capitatum & \\
\hline & & mentagrophytes & \\
\hline & & schoenleinii & \\
\hline & & verrucosum & \\
\hline \multirow{2}{*}{ Ascodesmiaceae } & \multirow{2}{*}{ Cephaliophora } & - & \multirow{2}{*}[186,196]{} \\
\hline & & irregularis & \\
\hline Basidiobolaceae & Basidiobolus & ranarum & {$[187]$} \\
\hline Bionectriaceae & Gliocladium & - & {$[186]$} \\
\hline \multirow{10}{*}{ Botryosphaeriaceae } & Auerswaldia & lignicola & {$[188]$} \\
\hline & \multirow{3}{*}{ Botryosphaeria } & - & \multirow{3}{*}[169,201]{} \\
\hline & & dothidea & \\
\hline & & rhodina & \\
\hline & Diplodia & - & {$[11]$} \\
\hline & Lasiodiplodia & theobromae & {$[196,207]$} \\
\hline & Macrophomina & phaseolina & {$[169,208]$} \\
\hline & Neoscytalidium & oculus & {$[203]$} \\
\hline & Neofusicoccum & mangiferae & [209] \\
\hline & Sphaeropsis & subglobosa & {$[175]$} \\
\hline \multirow{2}{*}{ Cephalothecaceae } & \multirow{2}{*}{ Phialemonium } & - & \multirow{2}{*}[202,210]{} \\
\hline & & curvatum & \\
\hline \multirow{2}{*}{ Ceratobsidiaceae } & \multirow{2}{*}{ Rhizoctonia } & - & \multirow{2}{*}[169,175]{} \\
\hline & & bataticola & \\
\hline \multirow{7}{*}{ Chaetomiaceae } & \multirow{3}{*}{ Chaetomium } & atrobrunneum & \\
\hline & & globosum & {$[47,168,191,214]$} \\
\hline & & strumarium & \\
\hline & Humicola & - & {$[12]$} \\
\hline & & heterothallica & \\
\hline & Thielavia & subthermophila & {$[169,204,205]$} \\
\hline & & tortuosa & \\
\hline Chaetosphaerellaceae & Diplosporium & - & {$[175]$} \\
\hline Chaetosnhaeriacege & Gongromeriza & - & {$[186]$} \\
\hline Chaetosphaeriaceae & Trichothecium & - & {$[186,206]$} \\
\hline Clavicinitaceae & Metarhizium & - & [220 - \\
\hline clavictpliaceae & Melarnizlum & anisopliae & \\
\hline Coniochaetaceae & Lecvthophora & - & {$\left[\begin{array}{lll}175 & 210\end{array}\right]$} \\
\hline Contocnaelaceae & Lecyinopnora & mutabilis & \\
\hline & Acrostalagmus & cinnabarensis & {$[28]$} \\
\hline Cordvcinitaceae & Beauveria & - & {$\left[\begin{array}{llll}47 & 191 & 229 & 230\end{array}\right]$} \\
\hline & Beauverla & bassiana & \\
\hline & Engyodontium & alba & {$[175]$} \\
\hline Corticiaceae & Sporotrichum & schenekii & {$[28,233]$} \\
\hline Corynesporascaceae & Corynespora & cassiicola & {$[251]$} \\
\hline & & - & \\
\hline & & albidus & \\
\hline Cryptococcaceae & Cryptococcus & curvatum & {$[212,222,252,253]$} \\
\hline & & laurentii & \\
\hline & & neoformans & \\
\hline
\end{tabular}




\begin{tabular}{|c|c|c|c|}
\hline Family & Genus & Species & References \\
\hline \multicolumn{4}{|c|}{ Fungal Keratitis } \\
\hline \multirow{2}{*}{ Cunninghamellaceae } & \multirow{2}{*}{ Cunninghamella } & - & \multirow{2}{*}[186,254]{} \\
\hline & & spinosum & \\
\hline Cystofilobasidiaceae & Guehomyces & pullulans & {$[222]$} \\
\hline \multirow{2}{*}{ Davidiellaceae } & Cladosporium & - & {$[115,247,257]$} \\
\hline & Davidiella & tassiana & {$[204]$} \\
\hline Debaryomycetaceae & Meyerozyma & caribbica & [259] \\
\hline Dermateaceae & Gloeosporium & fructigenum & [261] \\
\hline \multirow{2}{*}{ Diaporthaceae } & \multirow{2}{*}{ Phomopsis } & - & \multirow{2}{*}[262,263]{} \\
\hline & & phoenicicola & \\
\hline \multirow{6}{*}{ Didymellaceae } & \multirow{3}{*}{ Epicoccum } & - & \multirow{3}{*}[12,191,266]{} \\
\hline & & nigrum & \\
\hline & & sorghi & \\
\hline & \multirow{3}{*}{ Phoma } & - & \multirow{3}{*}[12,92,175]{} \\
\hline & & eupyrena & \\
\hline & & oculo-hominis & \\
\hline \multirow{3}{*}{ Dipodascaceae } & \multirow{2}{*}{ Geotrichum } & - & \multirow{2}{*}[175,210,281]{} \\
\hline & & candidum & \\
\hline & Magnusiomyces & capitatus & {$[282]$} \\
\hline \multirow{2}{*}{ Dothioraceae } & \multirow{2}{*}{ Aureobasidium } & - & \multirow{2}{*}[12,189,207,283]{} \\
\hline & & pullulans & \\
\hline Eremomycetaceae & Arthrographis & kalrae & {$[175,284]$} \\
\hline Gjaerumiaceae & Gjaerumia & minor & [285] \\
\hline \multirow{2}{*}{ Helotailes incertae sedis } & \multirow{2}{*}{ Scytalidium } & dimidiatum & \multirow{2}{*}[187,287]{} \\
\hline & & hyalinum & \\
\hline \multirow{17}{*}{ Herpotrichiellaceae } & \multirow{3}{*}{ Cladophialophora } & bantiana & \multirow{3}{*}[196,240,293]{} \\
\hline & & cladosporioides & \\
\hline & & carrionii & \\
\hline & \multirow{4}{*}{ Exophiala ${ }^{l}$} & dermatitidis & \\
\hline & & jeanselmei & \\
\hline & & jeanselmei var. dermatitidis & {$[115,146,154,175,289$ - 292] } \\
\hline & & jeanselmei var. jeanselmei & \\
\hline & & moniliae & \\
\hline & & phaeomuriformis & \\
\hline & & spinifera & \\
\hline & Fonsecaeg & compacta & {$\left[\begin{array}{llll}47 & 115 & 191 & 2941\end{array}\right]$} \\
\hline & Fonsecaea & pedrosoi & {$[4 /, 115,191,294]$} \\
\hline & & bubakii & \\
\hline & Phialophora & pedrosoi & {$[173,175,283]$} \\
\hline & & verrucosa & \\
\hline & Pullularia & - & [206] \\
\hline & Torula & - & [295] \\
\hline
\end{tabular}




\begin{tabular}{|c|c|c|c|}
\hline Family & Genus & Species & References \\
\hline \multicolumn{4}{|c|}{ Fungal Keratitis } \\
\hline \multirow{15}{*}{ Hypocreaceae } & \multirow{8}{*}{ Acremonium } & - & \multirow{8}{*}[127,166,175,191,193,247,296]{} \\
\hline & & atrogriseum & \\
\hline & & curvum & \\
\hline & & falciforme & \\
\hline & & kiliense & \\
\hline & & potronii & \\
\hline & & recifei & \\
\hline & & strictum & \\
\hline & Acrostalagmus & cinnabarensis & {$[28]$} \\
\hline & Gliocladium & - & {$[186]$} \\
\hline & Sepedonium & - & {$[270,283]$} \\
\hline & \multirow{4}{*}{ Trichoderma } & - & \multirow{4}{*}[28,146,193,222,240]{} \\
\hline & & hamatum & \\
\hline & & longibrachiatum & \\
\hline & & koningii & \\
\hline \multirow{2}{*}{ Hypocreales Incertae sedis } & Cephalosporium & - & {$[102,146]$} \\
\hline & Myrothecium & - & {$[175]$} \\
\hline Hyponectriaceae & Humicola & - & {$[12]$} \\
\hline \multirow{8}{*}{ Glomerellaceae } & \multirow{8}{*}{ Colletotrichum } & - & \multirow{8}{*}[41,189-195]{} \\
\hline & & atramentum & \\
\hline & & capsici & \\
\hline & & coccodes & \\
\hline & & dematium & \\
\hline & & gleosporiodes & \\
\hline & & graminicola & \\
\hline & & truncatum & \\
\hline & Glomerella & cingulata & {$[78,169]$} \\
\hline Gymnoascaceae & Gymnoascus & - & {$[197]$} \\
\hline Lagenidiaceae & Lagenidium & - & [198] \\
\hline \multirow{4}{*}{ Lasiosphaeriaceae } & Arthrinium & phaeospermum & {$[173,193]$} \\
\hline & Cladorrhinum & bulbilosum & {$[199,200]$} \\
\hline & Monotospora & - & [186] \\
\hline & Podospora & - & [169] \\
\hline \multirow{2}{*}{ Lichtheimiaceae } & \multirow{2}{*}{ Lichtheimia } & corymbifera & \multirow{2}{*}[193,204]{} \\
\hline & & ramosa & \\
\hline Lophiostomataceae & Tetrapola & - & [211] \\
\hline \multirow{3}{*}{ Malasseziaceae } & \multirow{3}{*}{ Malassezia } & - & \multirow{3}{*}[175,212,213]{} \\
\hline & & furfur & \\
\hline & & restricta & \\
\hline \multirow{2}{*}{ Massarinaceae } & \multirow{2}{*}{ Helminthosporium } & - & \multirow{2}{*}[10,90]{} \\
\hline & & maydis & \\
\hline Metacapnodiaceae & Hormiscium & - & {$[186]$} \\
\hline \multirow{6}{*}{ Microascaceae } & Doratomyces & - & {$[186]$} \\
\hline & Cephalotrichum & stemonitis & {$[175]$} \\
\hline & Graphium & eumorphum & [216] \\
\hline & Lophotrichus & - & {$[217]$} \\
\hline & \multirow{2}{*}{ Microascus } & brevicaulis & ] 101 190 \\
\hline & & gracilis & {$[29,191,218]$} \\
\hline
\end{tabular}




\begin{tabular}{|c|c|c|c|}
\hline Family & Genus & Species & References \\
\hline \multicolumn{4}{|c|}{ Fungal Keratitis } \\
\hline & Monosporium & - & [219] \\
\hline & Pseudallescheria & boydii & {$[47,191]$} \\
\hline & \multirow{3}{*}{ Scedosporium } & apiospermum & \multirow{3}{*}[174,196,220,221]{} \\
\hline & & boydii & \\
\hline & & prolificans & \\
\hline & \multirow{3}{*}{ Scopularisopsis } & - & \multirow{3}{*}[28,186,189,215]{} \\
\hline & & blochi & \\
\hline & & brevicaulis & \\
\hline & Wardomyces & - & [212] \\
\hline Montagnulaceae & Microsphaeropsis & olivacea & [225] \\
\hline \multirow{12}{*}{ Mucoraceae } & Chlamydoabsidia & padenii & {$[175]$} \\
\hline & \multirow{4}{*}{ Mucor } & - & \multirow{4}{*}[10,28,226,228]{} \\
\hline & & cornealis & \\
\hline & & racemosus & \\
\hline & & ramosissimus & \\
\hline & Rhizomucor & - & [29] \\
\hline & \multirow{5}{*}{ Rhizopus } & arrhizus & \multirow{5}{*}[28,173,191,193,219,226,227]{} \\
\hline & & nigricans & \\
\hline & & oryzae & \\
\hline & & parasiticus & \\
\hline & & stolonifer & \\
\hline & Zygorhynchus & - & {$[186]$} \\
\hline \multirow{3}{*}{ Mycosphaerellaceae } & Cercospora & - & {$[186]$} \\
\hline & Hormodendrum & - & {$[227,231]$} \\
\hline & Microcyclosporella & mali & {$[232]$} \\
\hline \multirow{27}{*}{ Nectriaceae } & Bactridium & - & {$[186]$} \\
\hline & \multirow{3}{*}{ Cylindrocarpon } & - & \multirow{3}{*}[11,191,247,365]{} \\
\hline & & destructans & \\
\hline & & lichenicola & \\
\hline & \multirow{23}{*}{ Fusarium } & - & \multirow{23}{*}{$127,146,172,175,193,199,234$ - 246] } \\
\hline & & aquaeductum & \\
\hline & & asiaticum & \\
\hline & & boothii & \\
\hline & & cerealis & \\
\hline & & chlamydosporum & \\
\hline & & culmorum & \\
\hline & & delphinoides & \\
\hline & & dimerum & \\
\hline & & episphaeria & \\
\hline & & equiseti & \\
\hline & & graminearum & \\
\hline & & incarnatum & \\
\hline & & incarnatum-equiseti & \\
\hline & & keratoplasticum & \\
\hline & & langsethiae & \\
\hline & & lateritium & \\
\hline & & lichenicola & \\
\hline & & moniliforme & \\
\hline & & musae & \\
\hline & & napiforme & \\
\hline & & nivale & \\
\hline & & nygamai & \\
\hline
\end{tabular}




\begin{tabular}{|c|c|c|c|}
\hline Family & Genus & Species & References \\
\hline \multicolumn{4}{|c|}{ Fungal Keratitis } \\
\hline \multirow{21}{*}{ Nectriaceae } & \multirow{16}{*}{ Fusarium } & oxysporum & \multirow{16}{*}{$127,146,172,175,193,199,234$ - 246] } \\
\hline & & penzigii & \\
\hline & & poae & \\
\hline & & polyphialidicum & \\
\hline & & proliferatum & \\
\hline & & pseudograminearum & \\
\hline & & roseum & \\
\hline & & sacchari & \\
\hline & & sambucinum & \\
\hline & & solani & \\
\hline & & sporotrichioides & \\
\hline & & subglutinans & \\
\hline & & temperatum & \\
\hline & & tricinctum & \\
\hline & & ventricosum & \\
\hline & & verticilloides & \\
\hline & Fusidium & terricola & {$[28]$} \\
\hline & Fusoma & - & {$[186]$} \\
\hline & \multirow{2}{*}{ Gibberalla } & avenacea $^{2}$ & \multirow{2}{*}[173,186,193]{} \\
\hline & & fujikuroi $^{3}$ & \\
\hline & Moniliaceae & - & [238] \\
\hline \multirow{3}{*}{ Neocosmospora } & keratoplastica & \multirow{3}{*}[204,248,249]{} & \\
\hline & rubicola & & \\
\hline & vasinfecta & & \\
\hline Sarcopodium & oculorum & [250] & \\
\hline Volutella & - & {$[112]$} & \\
\hline Niessliaceae & Stachybotrys & - & {$[186]$} \\
\hline \multirow{4}{*}{ Onygenaceae } & \multirow{3}{*}{ Chrysosporium } & - & \multirow{3}{*}[29,47,171,175]{} \\
\hline & & inops & \\
\hline & & parvum & \\
\hline & Coccidioides & immitis & {$[175]$} \\
\hline Ophiocordycipitaceae & Purpureocillium & lilacinum & {$[255]$} \\
\hline \multirow{2}{*}{ Ophiostomataceae } & \multirow{2}{*}{ Sporothrix } & pallida & \multirow{2}{*}[228,256]{} \\
\hline & & schenckii & \\
\hline Orbiliaceae & Arthrobotrys & oligospora & [258] \\
\hline Phaeosphaeriaceae & Tintelnotia & destructans & [260] \\
\hline Phycomycetaceae & Periconia & keratidis & {$[28]$} \\
\hline \multirow{3}{*}{ Plectosphaerellaceae } & Plectosporium & tabacinum & {$[265]$} \\
\hline & \multirow{2}{*}{ Verticillium } & - & \multirow{2}{*}[175,264]{} \\
\hline & & searrae & \\
\hline \multirow{11}{*}{ Pleosporaceae } & \multirow{7}{*}{ Alternaria } & alternata & \multirow{7}{*}{$171,191,204,270$ - 272] } \\
\hline & & chlamydospora & \\
\hline & & fusispora & \\
\hline & & infectoria & \\
\hline & & longipes & \\
\hline & & nees & \\
\hline & & tenuissima & \\
\hline & \multirow{4}{*}{ Bipolaris } & - & \\
\hline & & australiensis & {$\left[\begin{array}{llllll}146 & 196 & 257 & 273 & 274 & 283\end{array}\right.$} \\
\hline & & hawaiiensis & {$[140,190,251,215,214,203]$} \\
\hline & & oryzae & \\
\hline
\end{tabular}




\begin{tabular}{|c|c|c|c|}
\hline Family & Genus & Species & References \\
\hline \multicolumn{4}{|c|}{ Fungal Keratitis } \\
\hline \multirow{22}{*}{ Pleosporaceae } & & sorokiniana & \\
\hline & & spicifera & \\
\hline & Brachysporium & - & [275] \\
\hline & \multirow{3}{*}{ Cochliobolus } & - & \multirow{3}{*}[169,240]{} \\
\hline & & heterostrophus & \\
\hline & & spicifer & \\
\hline & \multirow{12}{*}{ Curvularia } & borreriae & \multirow{12}{*}{$34,111,115,171,175,191,249,267$ - 269] } \\
\hline & & brachyspora & \\
\hline & & clavata & \\
\hline & & crepinii & \\
\hline & & fallax & \\
\hline & & geniculata & \\
\hline & & lunata & \\
\hline & & pallescens & \\
\hline & & prasadii & \\
\hline & & senegalensis & \\
\hline & & spicifera & \\
\hline & & verruculosa & \\
\hline & Dichotomophthoropsis & nymphaearum & {$[249]$} \\
\hline & \multirow{2}{*}{ Drechslera } & - & \multirow{2}{*}[220,269]{} \\
\hline & & halodes & \\
\hline & Edenia & gomezpompae & [193] \\
\hline \multirow{6}{*}{ Exserohilum } & - & \multirow{6}{*}[175,190,199,207,276]{} & \\
\hline & longirostratum & & \\
\hline & mcginnisii & & \\
\hline & roseum & & \\
\hline & rostratum & & \\
\hline & solani & & \\
\hline Pithomyces & - & {$[34,212]$} & \\
\hline Pleospora & tarda & {$[146,240]$} & \\
\hline \multirow{2}{*}{ Pyrenochaeta } & - & \multirow{2}{*}[277,278]{} & \\
\hline & keratinophila & & \\
\hline Stemphylium & - & {$[279]$} & \\
\hline Ulocladium & atrum & {$[280]$} & \\
\hline Pleurotheciaceae & Phaeoisaria & clematidis & [79] \\
\hline Polyporaceae & Trametes & betulina & {$[80]$} \\
\hline Pythiaceae & Pythium & insidiosum & {$[47,81]$} \\
\hline \multirow{14}{*}{ Saccharomycetaceae } & Blastoschizomyces & capitatus & {$[175]$} \\
\hline & \multirow{13}{*}{ Candida } & albicans & \multirow{13}{*}[82-86,105,170,191,212,222,236,259,286]{} \\
\hline & & ciferrii & \\
\hline & & curvata & \\
\hline & & dubliniensis & \\
\hline & & famata & \\
\hline & & fermentati & \\
\hline & & glabrata & \\
\hline & & guilliermondii & \\
\hline & & krusei $^{4}$ & \\
\hline & & lusitaniae & \\
\hline & & lypolytica & \\
\hline & & orthopsilosis & \\
\hline & & parapsilosis & \\
\hline
\end{tabular}




\begin{tabular}{|c|c|c|c|}
\hline Family & Genus & Species & References \\
\hline \multicolumn{4}{|c|}{ Fungal Keratitis } \\
\hline & & pelliculosa & \multirow{6}{*}[18,48,53,61,68,125,163-169]{} \\
\hline & & rugosa & \\
\hline & & tropicalis & \\
\hline & & utilis & \\
\hline & & viswanathii & \\
\hline & & zeylanoides & \\
\hline & Saccharomyces & cerevisiae & [9] \\
\hline Schizophyllaceae & Schizophyllum & commune & [288] \\
\hline Schizoporaceae & Hyphodontia & - & [201] \\
\hline Sclerotiniaceae & Botrytis & - & {$[28]$} \\
\hline Sebacinaceae & Piriformospora & - & {$[146]$} \\
\hline Septobasidiaceae & Glenospora & graphii & {$[175]$} \\
\hline \multirow{2}{*}{ Sordariales incertae sedis } & \multirow{2}{*}{ Papulaspora } & - & \multirow{2}{*}[191,199]{} \\
\hline & & equi & \\
\hline \multirow{2}{*}{ Sordariaceae } & \multirow{2}{*}{ Neurospora } & - & \multirow{2}{*}[47,146]{} \\
\hline & & sitophila & \\
\hline \multirow{4}{*}{ Sporidiobolaceae } & \multirow{4}{*}{ Rhodotorula } & - & \multirow{4}{*}[270,283,308-311]{} \\
\hline & & glutinis & \\
\hline & & minuta & \\
\hline & & mucilaginosa & \\
\hline Sporocadaceae & Pseudopestalotiopsis & theae & [313] \\
\hline Sordariales & Pleurothecium & recurvatum & [315] \\
\hline Sympoventuriaceae & Verruconis & gallopava & {$[77]$} \\
\hline Syncephalastraceae & Syncephalastrum & - & [189] \\
\hline \multirow{2}{*}{ Togniniaceae } & \multirow{2}{*}{ Phaeoacremonium } & - & \multirow{2}{*}[316,317]{} \\
\hline & & parasiticum & \\
\hline Torulaceae & Torula & - & {$[12]$} \\
\hline \multirow{2}{*}{ Tetraplosphaeriaceae } & \multirow{2}{*}{ Tetraploa } & - & \multirow{2}{*}[102,175]{} \\
\hline & & aristata & \\
\hline Tremellaceae & Bulleromyces & - & [212] \\
\hline \multirow{19}{*}{ Trichocomaceae } & \multirow{19}{*}{ Aspergillus } & alternata & \multirow{19}{*}{$41,115,146,171,175,191,196,199,210,236,247,297$ - 302] } \\
\hline & & brasiliensis & \\
\hline & & cibarius & \\
\hline & & clavatus & \\
\hline & & fischerianus & \\
\hline & & flavipes & \\
\hline & & flavus & \\
\hline & & fumigatus & \\
\hline & & glaucus & \\
\hline & & janus & \\
\hline & & japonicus & \\
\hline & & nidulans & \\
\hline & & niger & \\
\hline & & niveus & \\
\hline & & nominus & \\
\hline & & ochraceus & \\
\hline & & oryzae & \\
\hline & & pseudotamarii & \\
\hline & & sydowii & \\
\hline
\end{tabular}




\begin{tabular}{|c|c|c|c|}
\hline Family & Genus & Species & References \\
\hline \multicolumn{4}{|c|}{ Fungal Keratitis } \\
\hline & & tamarii & \\
\hline & & terreus & \\
\hline & & tubingensis & \\
\hline & & versicolour & \\
\hline & & viridinutans & \\
\hline & & wentii & \\
\hline & Neosartorya & udagawae & {$[303]$} \\
\hline & \multirow{11}{*}{ Penicillium } & brocae & \multirow{11}{*}[9,102,146,175,210,240,306,307]{} \\
\hline & & canescens & \\
\hline & & chrysogenum & \\
\hline & & citrinum & \\
\hline & & crustaceum & \\
\hline & & expansum & \\
\hline & & implicatum & \\
\hline & & marneffei & \\
\hline & & notatum & \\
\hline & & piceum & \\
\hline & & spinulosum & \\
\hline & \multirow{4}{*}{ Paecilomyces } & - & \multirow{4}{*}[95,154,270,305]{} \\
\hline & & farinosus & \\
\hline & & lilacinus & \\
\hline & & variotti & \\
\hline & Sagenomella & keratitidis & [304] \\
\hline \multirow{3}{*}{ Trichosphaeriaceae } & Khuskia & - & [312] \\
\hline & \multirow{2}{*}{ Nigrospora } & - & \multirow{2}{*}[12,191,283]{} \\
\hline & & sphaerica & \\
\hline \multirow{6}{*}{ Trichosporonaceae } & \multirow{6}{*}{ Trichosporon } & anisopliae & \multirow{6}{*}[78,105,127,309,312,314]{} \\
\hline & & asahii & \\
\hline & & beigelii & \\
\hline & & capitatum & \\
\hline & & mucoides & \\
\hline & & rugosum & \\
\hline Tritirachiaceae & Tritirachium & oryzae & {$[175]$} \\
\hline \multirow{2}{*}{ Ustilaginaceae } & Rhodosporidium & toruloides & {$[175]$} \\
\hline & Ustilago & - & {$[175]$} \\
\hline Venturiaceae & Fusicladium & - & {$[186]$} \\
\hline \multirow{2}{*}{ Wickerhamomycetaceae } & Cyberlindnera & fabianii & [259] \\
\hline & Wickerhamomyces & anomalus & [318] \\
\hline \multirow{9}{*}{$n / a$} & Botryodiplodia & - & [366] \\
\hline & \multirow{3}{*}{ Dichotomophthoropsis } & - & \multirow{3}{*}[175,312]{} \\
\hline & & nymphearum & \\
\hline & & portulacae & \\
\hline & \multirow{2}{*}{ Mycelia } & - & \multirow{2}{*}[314,367]{} \\
\hline & & sterilia & \\
\hline & Ovadendron & sulphureo-ochraceum & {$[175]$} \\
\hline & \multirow{2}{*}{ Phaeotrichoconis } & crotalariae & {$[175]$} \\
\hline & & keratinophila & \\
\hline
\end{tabular}


Table 2. Diversity of Bacterial Keratitis [ ${ }^{1}$ Previously Comamonas] [ - ] Species not identified, New Pathogens in Bold.

\begin{tabular}{|c|c|c|c|}
\hline Family & Genus & Species & References \\
\hline \multicolumn{4}{|c|}{ Bacterial Keratitis } \\
\hline Acetobacteraceae & Roseomonas & - & [25] \\
\hline \multirow{2}{*}{ Actinomycetaceae } & \multirow{2}{*}{ Actinomyces } & bovis & \multirow{2}{*}[27,28]{} \\
\hline & & israelii & \\
\hline \multirow{3}{*}{ Aerococcaceae } & Abiotrophia & defectiva & {$[31]$} \\
\hline & \multirow{2}{*}{ Aerococcus } & - & \multirow{2}{*}[32,33]{} \\
\hline & & viridans & \\
\hline \multirow{2}{*}{ Aeromonadaceae } & \multirow{2}{*}{ Aeromonas } & - & \multirow{2}{*}[15,41,42]{} \\
\hline & & hydrophilia & \\
\hline \multirow{4}{*}{ Alcaligenaceae } & \multirow{3}{*}{ Achromobacter } & - & \multirow{3}{*}[33,42,51,52]{} \\
\hline & & denitrificans & \\
\hline & & xylosoxidans & \\
\hline & Alcaligenes & faecalis & [12] \\
\hline \multirow{9}{*}{ Bacillaceae } & \multirow{9}{*}{ Bacillus } & cefilius & \multirow{9}{*}[60-64]{} \\
\hline & & cereus & \\
\hline & & circulans & \\
\hline & & coagulans & \\
\hline & & firmus & \\
\hline & & licheniformis & \\
\hline & & megaterium & \\
\hline & & polymyxa & \\
\hline & & subtilis & \\
\hline Bacteroidaceae & Bacteroides & fragilis & {$[68]$} \\
\hline Bartonellaceae & Bartonella & henselae & {$[87]$} \\
\hline Brevibacteriaceae & Brevibacterium & - & [12] \\
\hline \multirow{3}{*}{ Brucellaceae } & Ochrobactrum & anthropi & [93] \\
\hline & \multirow{2}{*}{ Pasteurella } & canis & \multirow{2}{*}[49,94]{} \\
\hline & & multocida & \\
\hline \multirow{3}{*}{ Burkholderiaceae } & \multirow{3}{*}{ Burkholderia } & ambifaria & \multirow{3}{*}[47,96,97]{} \\
\hline & & cepacia & \\
\hline & & gladioli & \\
\hline Cardiobacteriaceae & Suttonella & indologenes & {$[34]$} \\
\hline Carnobacteriaceae & Dolosigranulum & pigrum & [101] \\
\hline Caulobacteraceae & Brevundimonas & diminuta & {$[108]$} \\
\hline \multirow{5}{*}{ Clostridiaceae } & \multirow{2}{*}{ Clostridium } & - & \multirow{2}{*}[60,110]{} \\
\hline & & perfringens & \\
\hline & \multirow{2}{*}{ Peptostreptococcus } & anaerobius & \multirow{2}{*}[68,111]{} \\
\hline & & micros & \\
\hline & Sarcina & - & [112] \\
\hline Comamonadaceae & Delftia $^{I}$ & acidovorans & {$[55,98]$} \\
\hline
\end{tabular}




\begin{tabular}{|c|c|c|c|}
\hline Family & Genus & Species & References \\
\hline \multicolumn{4}{|c|}{ Bacterial Keratitis } \\
\hline \multirow{13}{*}{ Corynebacteriaceae } & \multirow{13}{*}{ Corynebacterium } & accolens & \multirow{13}{*}[15,44,62,105,115-121]{} \\
\hline & & bovis & \\
\hline & & diptheriae & \\
\hline & & hofmannii & \\
\hline & & macginleyi & \\
\hline & & matruchotii & \\
\hline & & minutissimum & \\
\hline & & propinquum & \\
\hline & & pseudodiphtheriticum & \\
\hline & & pyogenes & \\
\hline & & striatum & \\
\hline & & urealyticum & \\
\hline & & ureicelerivorans & \\
\hline & & xerosis & \\
\hline & Arcanobacterium & haemolyticum & {$[117]$} \\
\hline \multirow{26}{*}{ Enterobacteriaceae } & \multirow{3}{*}{ Citrobacter } & diversus & \multirow{3}{*}[43,105,122,123]{} \\
\hline & & koseri & \\
\hline & & freundii & \\
\hline & \multirow{3}{*}{ Enterobacter } & aerogenes & \multirow{3}{*}[34,61,123,146]{} \\
\hline & & cloacae & \\
\hline & & gergoviae & \\
\hline & Escherichia & coli & {$[52,124]$} \\
\hline & Hafnia & alvei & [125] \\
\hline & \multirow{5}{*}{ Klebsiella } & ornithinolytica & \multirow{5}{*}[105,117,126,127,146]{} \\
\hline & & oxygenate & \\
\hline & & oxytoca & \\
\hline & & ozaenae & \\
\hline & & pneumoniae & \\
\hline & Morganella & morganii & {$[368]$} \\
\hline & \multirow{2}{*}{ Pantoea } & - & \multirow{2}{*}[65,129]{} \\
\hline & & agglomerans & \\
\hline & \multirow{4}{*}{ Proteus } & - & \multirow{4}{*}[52,126,130,368]{} \\
\hline & & mirabilis & \\
\hline & & rettgeri & \\
\hline & & vulgaris & \\
\hline & \multirow{4}{*}{ Providencia } & - & \multirow{4}{*}[33,104,131-133]{} \\
\hline & & alcalifaciens & \\
\hline & & rettgeri & \\
\hline & & stuartii & \\
\hline & \multirow{2}{*}{ Raoultella } & - & \multirow{2}{*}[33,134]{} \\
\hline & & ornithinolytica & \\
\hline Salmonella & - & [102] & \\
\hline \multirow{2}{*}{ Serratia } & liguefaciens & \multirow{2}{*}[52,135]{} & \\
\hline & marcescens & & \\
\hline \multirow{2}{*}{ Shigella } & flexneri & {$\left[\begin{array}{ll}52 & 136\end{array}\right]$} & \\
\hline & sonnei & {$[32,150]$} & \\
\hline & & faecalis & {$\left[\begin{array}{lll}23 & 20 & 1201\end{array}\right.$} \\
\hline Enterococcaceae & Enierococcus & faecium & {$[33,39,138]$} \\
\hline Eubacteriaceae & Eubacterium & aerofaciens & [143] \\
\hline
\end{tabular}




\begin{tabular}{|c|c|c|c|}
\hline Family & Genus & Species & References \\
\hline \multicolumn{4}{|c|}{ Bacterial Keratitis } \\
\hline \multirow{9}{*}{ Flavobacteriaceae } & \multirow{5}{*}{ Capnocytophaga } & - & \multirow{5}{*}[147-151]{} \\
\hline & & canimorsus & \\
\hline & & cynodegmi & \\
\hline & & ochracea & \\
\hline & & sputigena & \\
\hline & \multirow{2}{*}{ Chryseobacterium } & - & \multirow{2}{*}{ [152 - 154] } \\
\hline & & indologenes & \\
\hline & Elizabethkingia & meningoseptica & {$[34,155]$} \\
\hline & Flavobacterium & - & {$[52]$} \\
\hline Fusobacteriaceae & Fusobacterium & - & {$[157]$} \\
\hline Intrasporangiaceae & Ornithinimicrobium & pekingense & {$[160]$} \\
\hline Lactobacillaceae & Lactobacillus & - & {$[162]$} \\
\hline Leuconostocaceae & Leuconostoc & mesenteroides & {$[10]$} \\
\hline Listeriaceae & Listeria & monocytogenes & [26] \\
\hline \multirow{4}{*}{ Microbacteriaceae } & \multirow{2}{*}{ Microbacterium } & - & \multirow{2}{*}[29,30]{} \\
\hline & & oxydans & \\
\hline & \multirow{2}{*}{ Micrococcus } & - & \multirow{2}{*}[10,30,38,39]{} \\
\hline & & luteus & \\
\hline \multirow{8}{*}{ Micrococcaceae } & & tetragenus & \\
\hline & \multirow{5}{*}{ Kocuria } & koreensis & \multirow{5}{*}[10,34-37]{} \\
\hline & & kristinae & \\
\hline & & palustris & \\
\hline & & rosea & \\
\hline & & varians & \\
\hline & \multirow{2}{*}{ Rothia } & dentocariosa & \multirow{2}{*}[35,40]{} \\
\hline & & mucilaginosa & \\
\hline \multirow{10}{*}{ Moraxellaceae } & \multirow{7}{*}{ Acinetobacter } & baumannii & \multirow{7}{*}[43-48]{} \\
\hline & & calcoaceticus & \\
\hline & & calcoaceticus var. antitratus & \\
\hline & & haemolyticus & \\
\hline & & lwoffi & \\
\hline & & junii & \\
\hline & & schindleri & \\
\hline & & catarrhalis & \\
\hline & Moraxella & lacunata & {$[30,49,50]$} \\
\hline & & nonliquefaciens & \\
\hline \multirow{8}{*}{ Mycobacteriaceae } & & abscessus & \\
\hline & & asiaticum & \\
\hline & & aurum & \\
\hline & & avium & \\
\hline & & chelonae & \\
\hline & & flavescens & \\
\hline & & fotuitum & \\
\hline & Mucohasterium & gordonae & {$\left[\begin{array}{lll}57 & 52 & 501\end{array}\right.$} \\
\hline intracellulare & 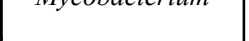 & & {$[4 /, 35-59]$} \\
\hline marinum & & & \\
\hline massiliense & & & \\
\hline mucogenicum & & & \\
\hline nonchromogenicum & & & \\
\hline szulgai & & & \\
\hline terrae & & & \\
\hline triviale & & & \\
\hline
\end{tabular}




\begin{tabular}{|c|c|c|c|}
\hline Family & Genus & Species & References \\
\hline \multicolumn{4}{|c|}{ Bacterial Keratitis } \\
\hline \multirow{6}{*}{ Neisseriaceae } & Eikenella & corrodens & {$[51]$} \\
\hline & \multirow{2}{*}{ Kingella } & denitrificans & \multirow{2}{*}[51,67]{} \\
\hline & & kingae & \\
\hline & \multirow{3}{*}{ Neisseria } & gonorrhoea & \multirow{3}{*}[13,65,66]{} \\
\hline & & meningitides & \\
\hline & & mucosa & \\
\hline \multirow{13}{*}{ Nocardiaceae } & \multirow{13}{*}{ Nocardia } & abscessus & \multirow{13}{*}[44,69-76]{} \\
\hline & & actinomycetes & \\
\hline & & amamiensis & \\
\hline & & araoensis & \\
\hline & & arthritidis & \\
\hline & & asiatica & \\
\hline & & asteroides & \\
\hline & & beijingensis & \\
\hline & & blacklockiae & \\
\hline & & brasiliensis & \\
\hline & & carnea & \\
\hline & & caviae & \\
\hline & & cyriacigeorgica & \\
\hline & \multirow{14}{*}{ Nocardia } & elegans & \multirow{14}{*}[237,291-298]{} \\
\hline & & farcinica & \\
\hline & & ignorata & \\
\hline & & levis & \\
\hline & & neocaledoniensis & \\
\hline & & nova & \\
\hline & & otitidiscaviarum & \\
\hline & & pneumoniae & \\
\hline & & pseudobrasiliensis & \\
\hline & & puris & \\
\hline & & rhamnosiphila & \\
\hline & & thailandica & \\
\hline & & transvalensis & \\
\hline & & wallacei & \\
\hline & Rhodococcus & equi & {$[77]$} \\
\hline \multirow{6}{*}{ Pasteurellaceae } & \multirow{2}{*}{ Aggregatibacter } & actinomycetemcomitans & \multirow{2}{*}[88,89]{} \\
\hline & & aphrophilus & \\
\hline & \multirow{4}{*}{ Haemophilus } & aegyticus & \multirow{4}{*}[41,44,46,90,91]{} \\
\hline & & haemoglobinophilus & \\
\hline & & influenza & \\
\hline & & parainfluenza & \\
\hline Peptococcaceae & Peptococcus & prevotii & {$[92]$} \\
\hline Porphyromonadaceae & Parabacteroides & distasonis & {$[95]$} \\
\hline \multirow{3}{*}{ Propionibacteriaceae } & \multirow{3}{*}{ Propionibacterium } & - & \multirow{3}{*}[1,68,98,99]{} \\
\hline & & acnes & \\
\hline & & granulosum & \\
\hline \multirow{3}{*}{ Prevotellaceae } & \multirow{3}{*}{ Prevotella } & - & \multirow{3}{*}[68,100]{} \\
\hline & & intermedia & \\
\hline & & melaninogenica & \\
\hline
\end{tabular}




\begin{tabular}{|c|c|c|c|}
\hline Family & Genus & Species & References \\
\hline \multicolumn{4}{|c|}{ Bacterial Keratitis } \\
\hline \multirow{13}{*}{ Pseudomonadaceae } & \multirow{4}{*}{ Azotobacter } & beijerinckii & \multirow{4}{*}[107]{} \\
\hline & & chroococcum & \\
\hline & & paspali & \\
\hline & & vinelandii & \\
\hline & \multirow{9}{*}{ Pseudomonas } & acidovorans & \multirow{9}{*}[30,33,38,52,102-106]{} \\
\hline & & aeruginosa & \\
\hline & & fluorescens & \\
\hline & & luteola & \\
\hline & & mesophilic & \\
\hline & & oryzihabitans & \\
\hline & & putida & \\
\hline & & pyocyaneus & \\
\hline & & stutzeri & \\
\hline Rhizobiaceae & Rhizobium & radiobacter & {$[109]$} \\
\hline Rhodobacteraceae & Paracoccus & yeei & [113] \\
\hline Rickettsiaceae & Rickettsia & conorii & {$[114]$} \\
\hline Sphingobacteriaceae & Sphingobacterium & spiritivorum & {$[30]$} \\
\hline \multirow{5}{*}{ Spirochaetaceae } & Borrelia & burgdorferi & [369] \\
\hline & Treponema & pallidum & {$[137]$} \\
\hline & \multirow{3}{*}{ Gemella } & - & \multirow{3}{*}[33,38,370]{} \\
\hline & & haemolysans & \\
\hline & & morbillorum & \\
\hline \multirow{10}{*}{ Staphylococcaceae } & \multirow{10}{*}{ Staphylococcus } & albus & \multirow{10}{*}{$10,15,23,34,39,43,46,52,60,139$ - 142] } \\
\hline & & aureus & \\
\hline & & auricularis & \\
\hline & & capitis & \\
\hline & & caprae & \\
\hline & & cohnii & \\
\hline & & epidermidis & \\
\hline & & haemolyticus & \\
\hline & & hominis & \\
\hline & & hyicus & \\
\hline \multirow{9}{*}{ Staphylococcaceae } & \multirow{9}{*}{ Staphylococcus } & intermedius & \multirow{9}{*}{$10,15,23,34,39,43,46,52,60,139$ - 142] } \\
\hline & & lentus & \\
\hline & & lugdunensis & \\
\hline & & $M R S A$ & \\
\hline & & pasteuri & \\
\hline & & saprophyticus & \\
\hline & & simulans & \\
\hline & & warneri & \\
\hline & & xylosus & \\
\hline
\end{tabular}




\begin{tabular}{|c|c|c|c|}
\hline Family & Genus & Species & References \\
\hline \multicolumn{4}{|c|}{ Bacterial Keratitis } \\
\hline \multirow{14}{*}{ Staphylococcaceae } & \multirow{14}{*}{ Staphylococcus } & acidominimus & \multirow{14}{*}{$33,41,46,47,49,60,64,65,117,126,144$ - 146] } \\
\hline & & agalactiae & \\
\hline & & anginosus & \\
\hline & & dysgalactiae & \\
\hline & & intermedius & \\
\hline & & mitis & \\
\hline & & morbillorum & \\
\hline & & oralis & \\
\hline & & parasanguis & \\
\hline & & pneumoniae & \\
\hline & & pyogenes & \\
\hline & & salivarius & \\
\hline & & sanguinis & \\
\hline & & viridans & \\
\hline \multirow{2}{*}{ Streptomycetaceae } & \multirow{2}{*}{ Streptomyces } & - & \multirow{2}{*}[131,156]{} \\
\hline & & thermocarboxydus & \\
\hline \multirow{3}{*}{ Tsukamurellaceae } & \multirow{3}{*}{ Tsukamurella } & hongkongensis & \multirow{3}{*}[158,159]{} \\
\hline & & spumae & \\
\hline & & tyrosinosolvens & \\
\hline \multirow{2}{*}{ Vibrionaceae } & \multirow{2}{*}{ Vibrio } & vulnificus & \multirow{2}{*}[43,161]{} \\
\hline & & parahaemolyticus & \\
\hline \multirow{2}{*}{ Xanthomonadaceae } & Stenotrophomonas & maltophilia & {$[46,98]$} \\
\hline & Xanthomonas & - & {$[163]$} \\
\hline \multirow{2}{*}{ Yersiniaceae } & \multirow{2}{*}{ Yersinia } & - & \multirow{2}{*}[43,164]{} \\
\hline & & pseudotuberculosis & \\
\hline
\end{tabular}

\section{REFERENCES}

[1] Hsiao CH, Sun CC, Yeh LK, et al. Shifting trends in bacterial keratitis in taiwan: A 10-year review in a tertiary-care hospital. Cornea 2016; 35(3): 313-7.

[http://dx.doi.org/10.1097/ICO.0000000000000734]

[PMID: 26764878]

[2] Austin A, Lietman T, Rose-Nussbaumer J. Update on the management of infectious keratitis. Ophthalmology 2017; 124(11): 1678-89. [http://dx.doi.org/10.1016/j.ophtha.2017.05.012] [PMID: 28942073]

[3] Watson S, Cabrera-Aguas M, Khoo P, Pratama R, Gatus BJ, Gulholm $\mathrm{T}$, et al. Keratitis antimicrobial resistance surveillance program, Sydney, Australia: 2016 Annual Report. Clin Exp Ophthalmol 2018. [PMID: 30047184]

[4] Bourcier T, Thomas F, Borderie V, Chaumeil C, Laroche L. Bacterial keratitis: Predisposing factors, clinical and microbiological review of 300 cases. Br J Ophthalmol 2003; 87(7): 834-8.

[http://dx.doi.org/10.1136/bjo.87.7.834] [PMID: 12812878]

[5] Tan SZ, Walkden A, Au L, et al. Twelve-year analysis of microbial keratitis trends at a UK tertiary hospital. Eye (Lond) 2017; 31(8): 1229-36.

[http://dx.doi.org/10.1038/eye.2017.55] [PMID: 28452995]

[6] Fernandes M, Vira D, Dey M, Tanzin T, Kumar N, Sharma S. Comparison between polymicrobial and fungal keratitis: Clinical features, risk factors, and outcome. Am J Ophthalmol 2015; 160(5): 873-881.e2.

[http://dx.doi.org/10.1016/j.ajo.2015.07.028] [PMID: 26210867]

[7] Karsten E, Watson SL, Foster LJR. Diversity of microbial species implicated in keratitis: A review. Open Ophthalmol J 2012; 6: 110-24. [http://dx.doi.org/10.2174/1874364101206010110] [PMID: 23248737]

[8] Gaujoux T, Borsali E, Gavrilov JC, Touzeau O, Goldschmidt P, Despiau MC, et al. Fungal keratitis caused by cylindrocarpon lichenicola. [French]. Journal Francais d'Ophtalmologie 2012; 35(5): 356e1-e5.

[9] Goldschmidt P, Degorge S, Benallaoua D, et al. New strategy for rapid diagnosis and characterization of keratomycosis. Ophthalmology 2012; 119(5): 945-50.

[http://dx.doi.org/10.1016/j.ophtha.2011.10.038] [PMID: 22342013]
[10] Lin L, Lan W, Lou B, et al. Genus distribution of bacteria and fungi associated with keratitis in a large eye center located in southern china. Ophthalmic Epidemiol 2017; 24(2): 90-6.

[http://dx.doi.org/10.1080/09286586.2016.1254250]

27960579]

[11] Laspina F, Samudio M, Cibils D, et al. Epidemiological characteristics of microbiological results on patients with infectious corneal ulcers: A 13-year survey in Paraguay. Graefes Arch Clin Exp Ophthalmol 2004; 242(3): 204-9.

[http://dx.doi.org/10.1007/s00417-003-0808-4] [PMID: 14760489]

[12] Gopinathan U, Sharma S, Garg P, Rao GN. Review of epidemiological features, microbiological diagnosis and treatment outcome of microbial keratitis: experience of over a decade. Indian J Ophthalmol 2009; 57(4): 273-9.

[http://dx.doi.org/10.4103/0301-4738.53051] [PMID: 19574694]

[13] Cariello AJ, Passos RM, Yu MC, Hofling-Lima AL. Microbial keratitis at a referral center in Brazil. Int Ophthalmol 2011; 31(3): 197-204.

[http://dx.doi.org/10.1007/s10792-011-9441-0] [PMID: 21448786]

[14] Kam KW, Yung W, Li GKH, Chen LJ, Young AL. Infectious keratitis and orthokeratology lens use: A systematic review. Infection 2017; 45(6): 727-35.

[http://dx.doi.org/10.1007/s15010-017-1023-2] [PMID: 28534320]

[15] Kaliamurthy J, Kalavathy CM, Parmar P, Nelson Jesudasan CA, Thomas PA. Spectrum of bacterial keratitis at a tertiary eye care centre in India. BioMed Res Int 2013; 2013181564

[http://dx.doi.org/10.1155/2013/181564] [PMID: 24066286]

[16] Al-Shehri A, Jastaneiah S, Wagoner MD. Changing trends in the clinical course and outcome of bacterial keratitis at King Khaled Eye Specialist Hospital. Int Ophthalmol 2009; 29(3): 143-52.

[http://dx.doi.org/10.1007/s10792-008-9206-6] [PMID: 18385946]

[17] Groups AE. Therapeutic guidelines: Antibiotic. 2014; Version 15.

[18] McDonald EMRF, Ram FS, Patel DV, McGhee CN. Topical antibiotics for the management of bacterial keratitis: An evidencebased review of high quality randomised controlled trials. $\mathrm{Br} \mathrm{J}$ Ophthalmol 2014; 98(11): 1470-7.

[http://dx.doi.org/10.1136/bjophthalmol-2013-304660] [PMID: 24729078] 
[19] Constantinou M, Daniell M, Snibson GR, Vu HT, Taylor HR. Clinical efficacy of moxifloxacin in the treatment of bacterial keratitis: A randomized clinical trial. Ophthalmology 2007; 114(9): 1622-9. [http://dx.doi.org/10.1016/j.ophtha.2006.12.011] [PMID: 17822972]

[20] O'Brien TPMM, Maguire MG, Fink NE, Alfonso E, McDonnell P. Efficacy of ofloxacin vs cefazolin and tobramycin in the therapy for bacterial keratitis. report from the bacterial keratitis study research group. Arch Ophthalmol 1995; 113(10): 1257-65.

[http://dx.doi.org/10.1001/archopht.1995.01100100045026] [PMID: 7575256]

[21] Hyndiuk RAER, Eiferman RA, Caldwell DR, et al. Comparison of ciprofloxacin ophthalmic solution $0.3 \%$ to fortified tobramycincefazolin in treating bacterial corneal ulcers. Ophthalmology 1996; 103(11): 1854-62.

[http://dx.doi.org/10.1016/S0161-6420(96)30416-8] [PMID: 8942881]

[22] Group. TOS. Ofloxacin monotherapy for the primary treatment of microbial keratitis: A double-masked, randomized, controlled trial with conventional dual therapy. Ophthalmology 1997; (104): 1902-9.

[23] Al-Dhaheri HSMD, Al-Tamimi MDMD, Khandekar RBMSPGD, Khan M, Stone DUMD. Ocular pathogens and antibiotic sensitivity in bacterial keratitis isolates at king khaled eye specialist hospital, 2011 to 2014. Cornea 2016; 35(6): 789-94.

[http://dx.doi.org/10.1097/ICO.0000000000000844] [PMID: 27078003]

[24] Ni N, Nam EM, Hammersmith KM, et al. Seasonal, geographic, and antimicrobial resistance patterns in microbial keratitis: 4-year experience in eastern Pennsylvania. Cornea 2015; 34(3): 296-302. [http://dx.doi.org/10.1097/ICO.0000000000000352] [PMID: 25603231]

[25] Tabin G, Danenhower C, Reardon D, Dimmig J, McCormick G. Opportunistic Roseomonas keratitis. Cornea 2001; 20(7): 772-3. [1]. [http://dx.doi.org/10.1097/00003226-200110000-00022] [PMID: 11588436]

[26] Altaie R, Fahy GT, Cormican M. Failure of Listeria monocytogenes keratitis to respond to topical ofloxacin. Cornea 2006; 25(7): 849-50. [http://dx.doi.org/10.1097/01.ico.0000230251.19847.3a] [PMID: 17068464]

[27] Karimian F, Feizi S, Nazari R, Zarin-Bakhsh P. Delayed-onset Actinomyces keratitis after laser in situ keratomileusis. Cornea 2008; 27(7): 843-6.

[http://dx.doi.org/10.1097/ICO.0b013e31816a624a] [PMID: 18650675]

[28] Keratomycosis GW. JAMA 1962; 179: 602-8

[http://dx.doi.org/10.1001/jama.1962.03050080014004] [PMID: 13898748]

[29] Ong HS, Fung SSM, Macleod D, Dart JKG, Tuft SJ, Burton MJ. Altered patterns of fungal keratitis at a london ophthalmic referral hospital: An eight-year retrospective observational study. Am J Ophthalmol 2016; 168: 227-36.

[http://dx.doi.org/10.1016/j.ajo.2016.05.021] [PMID: 27287820]

[30] Pandita A, Murphy C. Microbial keratitis in waikato, new zealand. Clin Exp Ophthalmol 2011; 39(5): 393-7.

[http://dx.doi.org/10.1111/j.1442-9071.2010.02480.x] [PMID: 21176039]

[31] Manderwad GP, Murthy SI, Motukupally SR. Postkeratoplasty keratitis caused by abiotrophia defectiva: An unusual cause of graft infection. Middle East Afr J Ophthalmol 2015; 22(3): 383-5. [http://dx.doi.org/10.4103/0974-9233.150631] [PMID: 26180481]

[32] Pachigolla G, Blomquist P, Cavanagh HD. Microbial keratitis pathogens and antibiotic susceptibilities: A 5-year review of cases at an urban county hospital in north Texas. Eye Contact Lens 2007; 33(1): $45-9$.

[http://dx.doi.org/10.1097/01.icl.0000234002.88643.d0] [PMID: 17224678]

[33] Oydanich M, Dingle TC, Hamula CL, Ghisa C, Asbell P. Retrospective report of antimicrobial susceptibility observed in bacterial pathogens isolated from ocular samples at Mount Sinai Hospital, 2010 to 2015. Antimicrob Resist Infect Control 2017; 6: 29. [http://dx.doi.org/10.1186/s13756-017-0185-0] [PMID: 28344783]

[34] Richards AD, Stewart CM, Karthik H, Petsoglou C. Microbial keratitis in indigenous Australians. Clin Exp Ophthalmol 2016; 44(3): 205-7. [http://dx.doi.org/10.1111/ceo.12643] [PMID: 26350024]

[35] Mattern RM, Ding J. Keratitis with Kocuria palustris and Rothia mucilaginosa in vitamin A deficiency. Case Rep Ophthalmol 2014; 5(1): $72-7$.

[http://dx.doi.org/10.1159/000360391] [PMID: 24707276]

[36] Pedro-Aguilar L, Ramirez-Miranda A, Bautista-de Lucio VM, Navas
A, Ortiz-Casas M, Graue-Hernandez EO. Epidemiology and outcomes of kocuria keratitis. Eye Contact Lens 2016; 42(5): e20-4. [http://dx.doi.org/10.1097/ICL.0000000000000173]

[PMID: 26222097]

[37] Inada N, Shoji J, Yamagami S. Atopic keratoconjunctivitis complicated by Kocuria koreensis keratitis: The first case. Allergy Asthma Clin Immunol 2017; 13: 6. [1]. [no pagination]. [6]. [http://dx.doi.org/10.1186/s13223-017-0178-9] [PMID: 28138334]

[38] Hernandez-Camarena JC, Graue-Hernandez EO, Ortiz-Casas M, et al. Trends in microbiological and antibiotic sensitivity patterns in infectious keratitis: 10-Year experience in mexico city. Cornea 2015; 34(7): 778-85.

[http://dx.doi.org/10.1097/ICO.0000000000000428] [PMID: 25811724]

[39] Faghri J, Zandi A, Peiman A, et al. Study on prevalence, antibiotic susceptibility, and tuf gene sequence-based genotyping of specieslevel of coagulase-negative staphylococcus isolated from keratitis caused by using soft contact lenses. Eye Contact Lens 2016; 42(2): $115-9$.

[http://dx.doi.org/10.1097/ICL.0000000000000066]

[PMID: 26629957]

[40] Morley AMS, Tuft SJ. Rothia dentocariosa isolated from a corneal ulcer. Cornea 2006; 25(9): 1128-9.

[http://dx.doi.org/10.1097/01.ico.0000226362.11431.81] [PMID: 17133072]

[41] Furlanetto RL, Andreo EG, Finotti IG, Arcieri ES, Ferreira MA, Rocha FJ. Epidemiology and etiologic diagnosis of infectious keratitis in Uberlandia, Brazil. Eur J Ophthalmol 2010; 20(3): 498-503. [http://dx.doi.org/10.1177/112067211002000312] [PMID: 20175055]

[42] Hoddenbach JG, Boekhoorn SS, Wubbels R, Vreugdenhil W, Van Rooij J, Geerards AJ. Clinical presentation and morbidity of contact lens-associated microbial keratitis: A retrospective study. Graefes Arch Clin Exp Ophthalmol 2014; 252(2): 299-306. [http://dx.doi.org/10.1007/s00417-013-2514-1] [PMID: 24281783]

[43] Song X, Xu L, Sun S, Zhao J, Xie L. Pediatric microbial keratitis: A tertiary hospital study. Eur J Ophthalmol 2012; 22(2): 136-41. [http://dx.doi.org/10.5301/EJO.2011.8338] [PMID: 21574163]

[44] Kunimoto DY, Sharma S, Garg P, Rao GN. In vitro susceptibility of bacterial keratitis pathogens to ciprofloxacin. Emerging resistance. Ophthalmology 1999; 106(1): 80-5.

[http://dx.doi.org/10.1016/S0161-6420(99)90008-8] [PMID: 9917785]

[45] Hung YT, Lee YT, Huang LJ, et al. Clinical characteristics of patients with Acinetobacter junii infection. J Microbiol Immunol Infect 2009; 42(1): 47-53

[PMID: 19424558]

[46] Schaftenaar E, Peters RPH, Baarsma GS, et al. Clinical and corneal microbial profile of infectious keratitis in a high HIV prevalence setting in rural South Africa. Eur J Clin Microbiol Infect Dis 2016; 35(9): 1403-9.

[http://dx.doi.org/10.1007/s10096-016-2677-x] [PMID: 27236644]

[47] Sirikul T, Prabriputaloong T, Smathivat A, Chuck RS, Vongthongsri A. Predisposing factors and etiologic diagnosis of ulcerative keratitis. Cornea 2008; 27(3): 283-7.

[http://dx.doi.org/10.1097/ICO.0b013e31815ca0bb] 18362653]

[48] Hongyok T, Leelaprute W. Corneal ulcer leading to evisceration or enucleation in a tertiary eye care center in Thailand: Clinical and microbiological characteristics. J Med Assoc Thai 2016; 99(Suppl. 2): S116-22.

[PMID: 27266225]

[49] Orlans HO, Hornby SJ, Bowler IC. In vitro antibiotic susceptibility patterns of bacterial keratitis isolates in Oxford, UK: A 10-year review. Eye (Lond) 2011; 25(4): 489-93.

[http://dx.doi.org/10.1038/eye.2010.231] [PMID: 21252952]

[50] Kowalski RP, Kowalski TA, Shanks RM, Romanowski EG, Karenchak LM, Mah FS. In vitro comparison of combination and monotherapy for the empiric and optimal coverage of bacterial keratitis based on incidence of infection. Cornea 2013; 32(6): 830-4. [http://dx.doi.org/10.1097/ICO.0b013e318268d6f4]

[PMID: 23132444

[51] Dahlgren MA, Lingappan A, Wilhelmus KR. The clinical diagnosis of microbial keratitis. Am J Ophthalmol 2007; 143(6): 940-4. [http://dx.doi.org/10.1016/j.ajo.2007.02.030] [PMID: 17408586]

[52] Goldstein MH, Kowalski RP, Gordon YJ. Emerging fluoroquinolone resistance in bacterial keratitis: a 5-year review. Ophthalmology 1999; 106(7): 1313-8.

[http://dx.doi.org/10.1016/S0161-6420(99)00716-2] [PMID: 
10406613]

[53] Skaat A, Zadok D, Goldich Y, et al. Riboflavin/UVA photochemical therapy for severe infectious keratitis. Eur J Ophthalmol 2014; 24(1): 21-8.

[http://dx.doi.org/10.5301/ejo.5000330] [PMID: 23873492]

[54] Honarvar B, Movahedan H, Mahmoodi M, Sheikholeslami FM, Farnia P. Mycobacterium aurum keratitis: An unusual etiology of a sightthreatening infection. Braz J Infect Dis 2012; 16(2): 204-8.

[http://dx.doi.org/10.1016/S1413-8670(12)70308-2]

[PMID: 22552468]

[55] Fong CF, Hu FR, Tseng CH, Wang IJ, Chen WL, Hou YC. Antibiotic susceptibility of bacterial isolates from bacterial keratitis cases in a university hospital in Taiwan. Am J Ophthalmol 2007; 144(5): 682-9. [http://dx.doi.org/10.1016/j.ajo.2007.06.038] [PMID: 17764652]

[56] Hung JH, Chang TC, Wu JJ, Lai CC, Huang FC, Huang YH. A cluster of Mycobacterium massiliense keratitis in foundry workers. Clinical Microbiology \& Infection 2016; 24(1): 386.e5-.e8 [http://dx.doi.org/10.1016/j.cmi.2015.12.014]

[57] Frueh BE, Dubuis O, Imesch $\mathrm{P}$, Böhnke $\mathrm{M}$, Bodmer $\mathrm{T}$. Mycobacterium szulgai keratitis. Arch Ophthalmol 2000; 118(8): 1123-4.

[http://dx.doi.org/10.1001/archopht.118.8.1123] [PMID: 10922211]

[58] Chu HS, Hu FR. Non-tuberculous mycobacterial keratitis. Clin Microbiol Infect 2013; 19(3): 221-6. [http://dx.doi.org/10.1111/1469-0691.12094] [PMID: 23211011]

[59] Moore MBNC, Newton C, Kaufman HE. Chronic keratitis caused by Mycobacterium gordonae. Am J Ophthalmol 1986; 102(4): 516-21. [http://dx.doi.org/10.1016/0002-9394(86)90083-8] [PMID: 3766669]

[60] Prokosch V, Gatzioufas Z, Thanos S, Stupp T. Microbiological findings and predisposing risk factors in corneal ulcers. Graefes Arch Clin Exp Ophthalmol 2012; 250(3): 369-74.

[http://dx.doi.org/10.1007/s00417-011-1722-9] [PMID: 22116498]

[61] Yu MC, Höfling-Lima AL, Furtado GH. Microbiological and epidemiological study of infectious keratitis in children and adolescents. Arq Bras Oftalmol 2016; 79(5): 289-93. [PMID: 27982205]

[62] Kunimoto DY, Sharma S, Garg P, Gopinathan U, Miller D, Rao GN. Corneal ulceration in the elderly in Hyderabad, south India. Br J Ophthalmol 2000; 84(1): 54-9.

[http://dx.doi.org/10.1136/bjo.84.1.54] [PMID: 10611100]

[63] Choudhuri KK, Sharma S, Garg P, Rao GN. Clinical and microbiological profile of Bacillus keratitis. Cornea 2000; 19(3): 301-6.

[http://dx.doi.org/10.1097/00003226-200005000-00009] [PMID: 10832688]

[64] Norina TJ, Raihan S, Bakiah S, Ezanee M, Liza-Sharmini AT, Wan Hazzabah WH. Microbial keratitis: Aetiological diagnosis and clinical features in patients admitted to Hospital Universiti Sains Malaysia. Singapore Med J 2008; 49(1): 67-71.

[PMID: 18204773]

[65] Ibrahim MM, Vanini R, Ibrahim FM, et al. Epidemiology and medical prediction of microbial keratitis in southeast Brazil. Arq Bras Oftalmol 2011; 74(1): 7-12.

[http://dx.doi.org/10.1590/S0004-27492011000100002] [PMID: 21670899]

[66] Scott IU, Flynn HW Jr, Feuer W, et al. Endophthalmitis associated with microbial keratitis. Ophthalmology 1996; 103(11): 1864-70. [http://dx.doi.org/10.1016/S0161-6420(96)30415-6] [PMID: 8942882]

[67] Yip H, Whiting M. Kingella kingae microbial keratitis in a human immunodeficiency virus patient with orthokeratology lens wear. Clin Exp Ophthalmol 2017; 45(4): 420-1.

[http://dx.doi.org/10.1111/ceo.12900] [PMID: 27943526]

[68] Tokman HB, İskeleli G, Dalar ZG, et al. Prevalence and antimicrobial susceptibilities of anaerobic bacteria isolated from perforated corneal ulcers by culture and multiplex PCR: an evaluation in cases with keratitis and endophthalmitis. Clin Lab 2014; 60(11): 1879-86. [http://dx.doi.org/10.7754/Clin.Lab.2014.131113] [PMID: 25648030]

[69] Shah P, Zhu D, Culbertson WW. Therapeutic femtosecond laserassisted lamellar keratectomy for multidrug-resistant nocardia keratitis. Cornea 2017; 36(11): 1429-31.

[http://dx.doi.org/10.1097/ICO.0000000000001318] [PMID: 28834821]

[70] Sun X, Deng S, Li R, et al. Distribution and shifting trends of bacterial keratitis in north China (1989-98). Br J Ophthalmol 2004; 88(2): 165-6. [1989-98].

[http://dx.doi.org/10.1136/bjo.2002.011205] [PMID: 14736761]

[71] Pandya VB, Petsoglou C. Nocardia transvalensis resistant to amikacin: an unusual cause of microbial keratitis. Cornea 2008; 27(9): 1082-5. [http://dx.doi.org/10.1097/ICO.0b013e3181783a20]

[PMID: 18812779]

[72] Lalitha P, Tiwari M, Prajna NV, Gilpin C, Prakash K, Srinivasan M. Nocardia keratitis: Species, drug sensitivities, and clinical correlation. Cornea 2007; 26(3): 255-9.

[http://dx.doi.org/10.1097/ICO.0b013e318033d853] [PMID: 17413948]

[73] Lalitha P. Nocardia keratitis. Curr Opin Ophthalmol 2009; 20(4): 318-23.

[http://dx.doi.org/10.1097/ICU.0b013e32832c3bcc]

[PMID: 19387343

[74] Lalitha P, Srinivasan M, Rajaraman R, et al. Nocardia keratitis: clinical course and effect of corticosteroids. Am J Ophthalmol 2012; 154(6): 934-939.e1

[http://dx.doi.org/10.1016/j.ajo.2012.06.001] [PMID: 22959881]

[75] Liu WL, Lai CC, Ko WC, et al. Clinical and microbiological characteristics of infections caused by various Nocardia species in Taiwan: A multicenter study from 1998 to 2010. Eur J Clin Microbiol Infect Dis $2011 ; 30(11): 1341-7$

[http://dx.doi.org/10.1007/s10096-011-1227-9] [PMID: 21461846]

[76] Reddy AK, Garg P, Kaur I. Spectrum and clinicomicrobiological profile of Nocardia keratitis caused by rare species of Nocardia identified by $16 \mathrm{~S}$ rRNA gene sequencing. Eye (Lond) 2010; 24(7): 1259-62.

[http://dx.doi.org/10.1038/eye.2009.299] [PMID: 19960036]

[77] Chan CC, Holland EJ. Infectious keratitis after Boston type 1 keratoprosthesis implantation. Cornea 2012; 31(10): 1128-34.

[http://dx.doi.org/10.1097/ICO.0b013e318245c02a]

[PMID: 22960647

[78] Embong Z, Wan Hitam WH, Yean CY, et al. Specific detection of fungal pathogens by $18 \mathrm{~S}$ rRNA gene PCR in microbial keratitis. BMC Ophthalmol 2008; 8: 7

[http://dx.doi.org/10.1186/1471-2415-8-7] [PMID: 18445283]

[79] Guarro J, Vieira LA, De Freitas D, et al. Phaeoisaria clematidis as a cause of keratomycosis. J Clin Microbiol 2000; 38(6): 2434-7. [PMID: 10835025]

[80] Hardin JS, Sutton DA, Wiederhold NP, Mele J, Goyal S. Fungal keratitis secondary to trametes betulina: A case report and review of literature. Mycopathologia 2017; 182(7-8): 755-9.

[http://dx.doi.org/10.1007/s11046-017-0128-6] [PMID: 28324243]

[81] Agarwal S, Iyer G, Srinivasan B, Agarwal M, Panchalam Sampath Kumar S, Therese LK. Clinical profile of pythium keratitis: Perioperative measures to reduce risk of recurrence. Br J Ophthalmol 2018; 102(2): 153-7

[http://dx.doi.org/10.1136/bjophthalmol-2017-310604] [PMID: 28903964]

[82] Chandra J, Mukherjee PK. Candida biofilms: Development, architecture, and resistance. Microbiol Spectr 2015; 3(4): 3. [4]. [http://dx.doi.org/10.1128/microbiolspec.MB-0020-2015] [PMID: 26350306]

[83] Deorukhkar S, Katiyar R, Saini S. Epidemiological features and laboratory results of bacterial and fungal keratitis: A five-year study at a rural tertiary-care hospital in western Maharashtra, India. Singapore Med J 2012; 53(4): 264-7.

[PMID: 22511050]

[84] Parentin F, Liberali T, Perissutti P. Polymicrobial keratomycosis in a three-year-old child. Ocul Immunol Inflamm 2006; 14(2): 129-31. [http://dx.doi.org/10.1080/09273940500328487] [PMID: 16597544]

[85] Kim MJ, Yu F, Aldave AJ. Microbial keratitis after Boston type I keratoprosthesis implantation: incidence, organisms, risk factors, and outcomes. Ophthalmology 2013; 120(11): 2209-16. [http://dx.doi.org/10.1016/j.ophtha.2013.05.001] [PMID: 23747162]

[86] Shih MH, Sheu MM, Chen HY, Lin SR. Fungal keratitis caused by Candida utilis-Case report. Kaohsiung J Med Sci 1999; 15(3): 171-4. [PMID: 10224841]

[87] Lohmann CPGB, Gabler B, Kroher G, Spiegel D, Linde HJ, Reisch U. Disciforme keratitis caused by Bartonella henselae: an unusual ocular complication in cat scratch disease. Eur J Ophthalmol 2000 10(3): $257-8$

[http://dx.doi.org/10.1177/112067210001000311] [PMID: 11071035]

88] Groden LRPS, Pascucci SE, Brinser JH. Haemophilus aphrophilus as a cause of crystalline keratopathy. Am J Ophthalmol 1987; 104(1): 89-90.

[http://dx.doi.org/10.1016/0002-9394(87)90303-5] [PMID: 3605289]

[89] Hong J, Xu J, Cao W, Ji J, Sun X. Actinobacillus actinomycetemcomitans keratitis after glaucoma infiltration surgery: A 
clinical report and literature review. Medicine (Baltimore) 2016; 95(3)e2608 [United States].

[http://dx.doi.org/10.1097/MD.0000000000002608] [PMID: 26817919]

[90] Panda A, Satpathy G, Nayak N, Kumar S, Kumar A. Demographic pattern, predisposing factors and management of ulcerative keratitis: evaluation of one thousand unilateral cases at a tertiary care centre. Clin Exp Ophthalmol 2007; 35(1): 44-50.

[http://dx.doi.org/10.1111/j.1442-9071.2007.01417.x] [PMID: 17300570]

[91] Das S, Sharma S, Priyadarshini O, Sahu SK, Kar S, Vemuganti GK. Association between culture results of corneal scrapings and culture and histopathology results of corneal tissues in therapeutic keratoplasty. Cornea 2011; 30(9): 1003-6.

[http://dx.doi.org/10.1097/ICO.0b013e318206ccfl] [PMID: 21464704]

[92] Carmichael TR, Wolpert M, Koornhof HJ. Corneal ulceration at an urban African hospital. Br J Ophthalmol 1985; 69(12): 920-6. [http://dx.doi.org/10.1136/bjo.69.12.920] [PMID: 3936534]

[93] Venkateswaran NW, Rachel AF. Hindman, Holly B. Ochrobactrum anthropi keratitis with focal descemet's membrane detachment and intracorneal hypopyon. Case Rep Ophthalmol Med 2016.

[http://dx.doi.org/10.1155/2016/4502105]

[94] Shah A, Talati M, Mauger T. Medical and surgical management of Pasteurella canis infectious keratitis. IDCases 2017; 9: 42-4. [http://dx.doi.org/10.1016/j.idcr.2017.05.012] [PMID: 28660128]

[95] Ali TK, Amescua G, Miller D, et al. Contact-Lens-Associated purpureocillium keratitis: Risk factors, microbiologic characteristics, clinical course, and outcomes. Semin Ophthalmol 2017; 32(2): 157-62. [http://dx.doi.org/10.3109/08820538.2015.1011342] [PMID: 25723808]

[96] Ritterband D, Shah M, Cohen K, Lawrence J, Seedor J. Burkholderia gladioli keratitis associated with consecutive recurrent endophthalmitis. Cornea 2002; 21(6): 602-3.

[http://dx.doi.org/10.1097/00003226-200208000-00014] [PMID: 12131039]

[97] Matoba AY. Polymicrobial keratitis secondary to Burkholderia ambifaria, enterococcus, and staphylococcus aureus in a patient with herpetic stromal keratitis. Am J Ophthalmol 2003; 136(4): 748-9. [http://dx.doi.org/10.1016/S0002-9394(03)00395-7]

[PMID: 14516823]

[98] Rossetto JD, Cavuoto KM, Osigian CJ, et al. Paediatric infectious keratitis: A case series of 107 children presenting to a tertiary referral centre. Br J Ophthalmol 2017; 101(11): 1488-92.

[http://dx.doi.org/10.1136/bjophthalmol-2016-310119] [PMID: 28298316]

[99] Lim SA, Na KS, Joo CK. Clinical features of infectious keratitis caused by propionibacterium acnes. Eye Contact Lens 2017; 43(5): 330-3.

[http://dx.doi.org/10.1097/ICL.0000000000000281] [PMID: 27203796]

[100] Toshida H, Kogure N, Inoue N, Murakami A. Trends in microbial keratitis in Japan. Eye \& Contact Lens: Science \&. Clin Pract 2007; 33(2): 70-3.

[101] M. Sampo OG. D. Cadiou, E. Trichet, L. Hoffart and M. Drancourt. Dolosigranulum pigrum keratitis: A three-case series. BMC Ophthalmol 2013; 13.

[102] Sony P, Sharma N, Vajpayee RB, Ray M. Therapeutic keratoplasty for infectious keratitis: A review of the literature. CLAO J 2002; 28(3): 111-8. [PMID: 12144228]

[103] Vieira AC, Pereira T, de Freitas D. Late-onset infections after LASIK. J Refract Surg 2008; 24(4): 411-3.

[http://dx.doi.org/10.3928/1081597X-20080401-16] [PMID: 18500093]

[104] Watt KG, Swarbrick HA. Trends in microbial keratitis associated with orthokeratology. Eye Contact Lens 2007; 33(6 Pt 2): 373-7. [http://dx.doi.org/10.1097/ICL.0b013e318157cd8d] [PMID: 17975424]

[105] Alkatan H, Athmanathan S, Canites CC. Incidence and microbiological profile of mycotic keratitis in a tertiary care eye hospital: A retrospective analysis. Saudi J Ophthalmol 2012; 26(2): 217-21.

[http://dx.doi.org/10.1016/j.sjopt.2011.11.005] [PMID: 23960995]

[106] Nascimento H, Watanabe A, Vieira ACC, et al. Detection of herpes simplex-1 and -2 and varicella zoster virus by quantitative real-time polymerase chain reaction in corneas from patients with bacterial keratitis. Arq Bras Oftalmol 2017; 80(2): 84-7. [PMID: 28591279]

[107] Liesegang TJ, Jones DR, Robinson NM. Azotobacter keratitis. Arch Ophthalmol 1981; 99(9): 1587-90.

[http://dx.doi.org/10.1001/archopht.1981.03930020461011] [PMID: 7283809]

[108] Pandit RT. Brevundimonas diminuta keratitis. Eye Contact Lens 2012; 38(1): 63-5.

[http://dx.doi.org/10.1097/ICL.0b013e31821c04f7] [PMID: 21617535]

[109] Barker NH, Thompson JM, Mullen MG, et al. Rhizobium radiobacter: A recently recognized cause of bacterial keratitis. Cornea 2016; 35(5): 679-82.

[http://dx.doi.org/10.1097/ICO.0000000000000780] [PMID: 26938327]

[110] Amer Awan M, Reeks G, Rahman MQ, Butcher I, Ramaesh K. The patterns of in vitro antimicrobial susceptibility and resistance of bacterial keratitis isolates in Glasgow, United Kingdom. Clin Exp Optom 2010; 93(5): 354-9.

[http://dx.doi.org/10.1111/j.1444-0938.2010.00511.x] [PMID: 20831515]

[111] Wilhelmus KRJD, Jones DB. Curvularia keratitis. Trans Am Ophthalmol Soc 2001; 99: 111-30. [111-30]. [PMID: 11797300]

[112] Liesegang TJFR, Forster RK. Spectrum of microbial keratitis in South Florida. Am J Ophthalmol 1980; 90(1): 38-47.

[http://dx.doi.org/10.1016/S0002-9394(14)75075-5] [PMID: 7395957]

[113] Courjaret JC, Drancourt M, Hoffart L. Paracoccus yeei keratitis in a contact lens wearer. Eye Contact Lens 2014; 40(3): e21-2.

[http://dx.doi.org/10.1097/ICL.0b013e31829e8fc7] [PMID: 24045834]

[114] Alio J, Ruiz-Beltran R, Herrera I, Artola A, Ruiz-Moreno JM. Rickettsial keratitis in a case of Mediterranean spotted fever. Eur J Ophthalmol 1992; 2(1): 41-3

[http://dx.doi.org/10.1177/112067219200200111] [PMID: 1638167]

[115] Bhadange Y, Sharma S, Das S, Sahu SK. Role of liquid culture media in the laboratory diagnosis of microbial keratitis. Am J Ophthalmol 2013; 156(4): 745-51.

[http://dx.doi.org/10.1016/j.ajo.2013.05.035] [PMID: 23916751]

[116] Das S, Rao AS, Sahu SK, Sharma S. Corynebacterium spp as causative agents of microbial keratitis. Br J Ophthalmol 2016; 100(7): 939-43.

[http://dx.doi.org/10.1136/bjophthalmol-2015-306749]

[PMID: 26567025]

[117] Ly CN, Pham JN, Badenoch PR, et al. Bacteria commonly isolated from keratitis specimens retain antibiotic susceptibility to fluoroquinolones and gentamicin plus cephalothin. Clin Exp Ophthalmol 2006; 34(1): 44-50.

[http://dx.doi.org/10.1111/j.1442-9071.2006.01143.x] [PMID: 16451258]

[118] Basak SK, Basak S, Mohanta A, Bhowmick A. Epidemiological and microbiological diagnosis of suppurative keratitis in Gangetic West Bengal, eastern India. Indian J Ophthalmol 2005; 53(1): 17-22. [http://dx.doi.org/10.4103/0301-4738.15280] [PMID: 15829742]

[119] Fernandes M, Sharma S. Polymicrobial and microsporidial keratitis in a patient using Boston scleral contact lens for Sjogren's syndrome and ocular cicatricial pemphigoid. Cont Lens Anterior Eye 2013; 36(2): 95-7.

[http://dx.doi.org/10.1016/j.clae.2012.10.082] [PMID: 23123433]

[120] Todokoro D, Eguchi H, Yamada N, Sodeyama H, Hosoya R, Kishi S. Contact Lens-Related Infectious Keratitis with White Plaque Formation Caused by Corynebacterium propinquum. J Clin Microbiol 2015; 53(9): 3092-5.

[http://dx.doi.org/10.1128/JCM.00899-15] [PMID: 26179302]

[121] Battaini A, La Scola B, Yin GHW, Hoffart L, Drancourt M. Amebaborne "Attilina massiliensis" Keratitis, France. Emerg Infect Dis 2018; 24(2): 387-9.

[http://dx.doi.org/10.3201/eid2402.170541] [PMID: 29350163]

[122] Goold LA, Warrier SK, Wittles NK, Nathan F. Microbial keratitis secondary to infection with Citrobacter koseri. Cornea 2010; 29(4): 479 .

[http://dx.doi.org/10.1097/ICO.0b013e3181ba00e9]

[PMID: 20164753.

[123] Hooi SH, Hooi ST. Culture-proven bacterial keratitis in a Malaysian general hospital. Med J Malaysia 2005; 60(5): 614-23. [PMID: 16515113]

[124] Micelli Ferrari T, Leozappa M, Lorusso M, Epifani E, Micelli Ferrari L. Escherichia coli keratitis treated with ultraviolet $\mathrm{A} /$ riboflavin corneal cross-linking: A case report. Eur J Ophthalmol 2009; 19(2): 
295-7.

[http://dx.doi.org/10.1177/112067210901900221] [PMID: 19253251]

[125] Coster DJ, Badenoch PR. Host, microbial, and pharmacological factors affecting the outcome of suppurative keratitis. Br J Ophthalmol 1987; 71(2): 96-101

[http://dx.doi.org/10.1136/bjo.71.2.96] [PMID: 3103672]

[126] Chawla B, Agarwal P, Tandon R, et al. In vitro susceptibility of bacterial keratitis isolates to fourth-generation fluoroquinolones. Eur J Ophthalmol 2010; 20(2): 300-5.

[http://dx.doi.org/10.1177/112067211002000207] [PMID: 19924668]

[127] Stapleton F, Naduvilath T, Keay L, et al. Risk factors and causative organisms in microbial keratitis in daily disposable contact lens wear. PLoS One 2017; 12(8)e0181343 [Electronic Resource].

[http://dx.doi.org/10.1371/journal.pone.0181343] [PMID: 28813424]

[128] Willcox MD, Holden BA. Contact lens related corneal infections. Biosci Rep 2001; 21(4): 445-61.

[http://dx.doi.org/10.1023/A:1017991709846] [PMID: 11900321]

[129] Sueke H, Kaye S, Neal T, et al. Minimum inhibitory concentrations of standard and novel antimicrobials for isolates from bacterial keratitis. Invest Ophthalmol Vis Sci 2010; 51(5): 2519-24.

[http://dx.doi.org/10.1167/iovs.09-4638] [PMID: 20019362]

[130] John D, Daniel E. Infectious keratitis in leprosy. Br J Ophthalmol 1999; 83(2): 173-6.

[http://dx.doi.org/10.1136/bjo.83.2.173] [PMID: 10396193]

[131] Rahimi F, Hashemian MN, Khosravi A, Moradi G, Bamdad S. Bacterial keratitis in a tertiary eye centre in Iran: A retrospective study. Middle East Afr J Ophthalmol 2015; 22(2): 238-44. [http://dx.doi.org/10.4103/0974-9233.151870] [PMID: 25949085]

[132] Choi W, Ji YS, Yoon KC. A case of bilateral keratitis caused by Providencia alcalifaciens: A rarely encountered ocular pathogen. Int Ophthalmol 2017; 1-4.

[PMID: 28516225]

[133] Koreishi AF, Schechter BA, Karp CL. Ocular infections caused by Providencia rettgeri. Ophthalmology 2006; 113(8): 1463-6. [http://dx.doi.org/10.1016/j.ophtha.2006.03.047] [PMID: 16797710]

[134] Eguchi H, Hotta F, Kuwahara T, Nakayama-Imaohji H, Kusaka S, Shimomura Y. Acute keratoconjunctivitis due to contamination of contact lens care solution with histamine-producing Raoultella species: A case report. Medicine (Baltimore) 2017; 96(50)e9310 [United States].

[http://dx.doi.org/10.1097/MD.0000000000009310] [PMID: 29390396]

[135] Stapleton F, Keay LJ, Sanfilippo PG, Katiyar S, Edwards KP, Naduvilath T. Relationship between climate, disease severity, and causative organism for contact lens-associated microbial keratitis in Australia. Am J Ophthalmol 2007; 144(5): 690-8.

[http://dx.doi.org/10.1016/j.ajo.2007.06.037] [PMID: 17727808]

[136] Muytjens HL, Eggink CA, Dijkman FC, Bakkers JM, Melchers WJ. Keratitis due to Shigella flexneri. J Clin Microbiol 2006; 44(6): 2291-4.

[http://dx.doi.org/10.1128/JCM.00481-06] [PMID: 16757643]

[137] P. Ruusuvaara KS. T. Kivelä. Syphilitic Interstitial keratitis with bilateral funnel-shaped iridocorneal adhesions. Eur J Ophthalmol 1996; 6(1): 6-10.

[http://dx.doi.org/10.1177/112067219600600102] [PMID: 8744842]

[138] Callegan MC, Ramirez R, Kane ST, Cochran DC, Jensen H. Antibacterial activity of the fourth-generation fluoroquinolones gatifloxacin and moxifloxacin against ocular pathogens. Adv Ther 2003; 20(5): 246-52.

[http://dx.doi.org/10.1007/BF02849853] [PMID: 14964344]

[139] Magli A, Forte R, Rombetto L, Carelli R. Bilateral methicillinresistant Staphylococcus aureus keratitis following hyperopic photorefractive surgery. Int Ophthalmol 2012; 32(1): 47-9. [http://dx.doi.org/10.1007/s10792-011-9505-1] [PMID: 22215418]

[140] Hong J, Chen J, Sun X, et al. Paediatric bacterial keratitis cases in Shanghai: Microbiological profile, antibiotic susceptibility and visual outcomes. Eye (Lond) 2012; 26(12): 1571-8.

[http://dx.doi.org/10.1038/eye.2012.210] [PMID: 23079751]

[141] P. Manikandan MB, R. Revathy, R. K John, K. Narendran, V. Narendran speciation of coagulase negative staphylococcus causing bacterial keratitis. 2005; 53: p. (1)59-60.

[142] Schaftenaar E, van Gorp EC, Meenken C, et al. Ocular infections in sub-Saharan Africa in the context of high HIV prevalence. Trop Med Int Health 2014; 19(9): 1003-14.

[http://dx.doi.org/10.1111/tmi.12350] [PMID: 25039335]

[143] Pate JC, Jones DB, Wilhelmus KR. Prevalence and spectrum of bacterial co-infection during fungal keratitis. Br J Ophthalmol 2006;
90(3): 289-92.

[http://dx.doi.org/10.1136/bjo.2005.081869] [PMID: 16488946]

[144] Chidambaram JD, Kannambath S, Srikanthi P, et al. Persistence of Innate Immune Pathways in Late Stage Human Bacterial and Fungal Keratitis: Results from a Comparative Transcriptome Analysis. Front Cell Infect Microbiol 2017; 7: 193.

[http://dx.doi.org/10.3389/fcimb.2017.00193] [PMID: 28573109]

[145] Henry CR, Flynn HW Jr, Miller D, Forster RK, Alfonso EC. Infectious keratitis progressing to endophthalmitis: A 15-year study of microbiology, associated factors, and clinical outcomes. Ophthalmology 2012; 119(12): 2443-9.

[http://dx.doi.org/10.1016/j.ophtha.2012.06.030] [PMID: 22858123]

[146] Sung MS, Choi W, You IC, Yoon KC. Factors affecting treatment outcome of graft infection following penetrating keratoplasty. Korean J Ophthalmol 2015; 29(5): 301-8.

[http://dx.doi.org/10.3341/kjo.2015.29.5.301] [PMID: 26457035]

[147] Paton BG, Ormerod LD, Peppe J, Kenyon KR. Evidence for a feline reservoir for dysgonic fermenter 2 keratitis. J Clin Microbiol 1988; 26(11): 2439-40.

[PMID: 3235673]

[148] Roussel TJ, Osato MS, Wilhelmus KR. Capnocytophaga keratitis. Br J Ophthalmol 1985; 69(3): 187-8.

[http://dx.doi.org/10.1136/bjo.69.3.187] [PMID: 3978064]

[149] Ormerod LD, Foster CS, Paton BG, Haaf J, Baker AS. Ocular Capnocytophaga infection in an edentulous, immunocompetent host. Cornea 1988; 7(3): 218-22

[http://dx.doi.org/10.1097/00003226-198803000-00008] [PMID: 3168490]

[150] Wright TM, Afshari NA. Microbial keratitis following corneal transplantation. Am J Ophthalmol 2006; 142(6): 1061-2. [http://dx.doi.org/10.1016/j.ajo.2006.06.051] [PMID: 17157593]

[151] Seitzman GD, Thulasi P, Hinterwirth A, Chen C, Shantha J, Doan T Capnocytophaga keratitis: Clinical presentation and use of metagenomic deep sequencing for diagnosis. Cornea 2019; 38(2): 246-8. [PMID: 30346341]

[152] Su PY, Hu FR, Chen YM, Han JH, Chen WL. Dendritiform cells found in central cornea by in-vivo confocal microscopy in a patient with mixed bacterial keratitis. Ocul Immunol Inflamm 2006; 14(4): 241-4.

[http://dx.doi.org/10.1080/09273940600732398] [PMID: 16911987]

[153] Ramos-Esteban JC, Bamba S, Jeng BH. Treatment of multidrugresistant Flavobacterium indologenes keratitis with trimethoprimsulfamethoxazole. Cornea 2008; 27(9): 1074-6.

[http://dx.doi.org/10.1097/ICO.0b013e318176189e] [PMID: 18812776]

[154] Passos RM, Cariello AJ, Yu MC, Höfling-Lima AL. Microbial keratitis in the elderly: A 32-year review. Arq Bras Oftalmol 2010; 73(4): 315-9.

[http://dx.doi.org/10.1590/S0004-27492010000400002] [PMID: 20944931]

[155] Erdem E, Abdurrahmanoglu S, Kibar F, Yagmur M, Koksal F, Ersoz R. Posttraumatic keratitis caused by Elizabethkingia meningosepticum. Eye Contact Lens 2013; 39(5): 361-3.

[http://dx.doi.org/10.1097/ICL.0b013e318291d171] 23719581]

[156] Kawakami H, Inuzuka H, Mochizuki K, et al. Case of keratitis caused by Streptomyces thermocarboxydus. J Infect Chemother 2014; 20(1): 57-60.

[http://dx.doi.org/10.1016/j.jiac.2013.11.001] [PMID: 24462427]

[157] Fröhlich SJ, Miño de Kaspar H, Grasbon T, Möhring C, Klauss V, Kampik A. Bacterial keratitis. Microbiological analysis as a principle for therapeutic recommendations. Ophthalmologe 1999; 96(7): 459-64. [PMID: 10479898]

[158] Liu CY, Lai CC, Lee MR, et al. Clinical characteristics of infections caused by Tsukamurella spp. and antimicrobial susceptibilities of the isolates. Int J Antimicrob Agents 2011; 38(6): 534-7.

[http://dx.doi.org/10.1016/j.ijantimicag.2011.07.018] [PMID: 22014886]

[159] Teng JLL, Tang Y, Wong SS, et al. Tsukamurella hongkongensis sp. nov. and Tsukamurella sinensis sp. nov., isolated from patients with keratitis, catheter-related bacteraemia and conjunctivitis. Int J Syst Evol Microbiol 2016; 66(1): 391-7.

[http://dx.doi.org/10.1099/ijsem.0.000733] [PMID: 26530900]

[160] Borsali E, Le Bouter A, Abdiche G, et al. Ornithinimicrobium pekingense ocular infection. Med Mal Infect 2011; 41(6): 345-6. [French]. 
[http://dx.doi.org/10.1016/j.medmal.2010.12.015] [PMID: 21440391]

[161] Penland RLBM, Boniuk M, Wilhelmus KR. Vibrio ocular infections on the U.S. Gulf Coast. Cornea 2000; 19(1): 26-9.

[http://dx.doi.org/10.1097/00003226-200001000-00006] [PMID: 10632004]

[162] Tam ALC, Côté E, Saldanha M, Lichtinger A, Slomovic AR. Bacterial keratitis in toronto: A 16-Year review of the microorganisms isolated and the resistance patterns observed. Cornea 2017; 36(12): 1528-34. [http://dx.doi.org/10.1097/ICO.0000000000001390] [PMID: 28938380]

[163] Miedziak AIMM, Miller MR, Rapuano CJ, Laibson PR, Cohen EJ. Risk factors in microbial keratitis leading to penetrating keratoplasty. Ophthalmology 1999; 106(6): 1166-70.

[http://dx.doi.org/10.1016/S0161-6420(99)90250-6] [PMID: 10366087]

[164] Narsani AK, Jatoi SM, Khanzada MA, Lohana MK. Etiological diagnosis of microbial keratitis. J Coll Physicians Surg Pak 2010; 20(9): 604-7.

[PMID: 20810053]

[165] Reynolds G, Campbell L, Monroe-Williams TD, Heslop O. Microbiological profile of mycotic eye infections at a tertiary care institution in the caribbean: A retrospective analysis. International Journal of Infectious Diseases 2016; 316-7.

[http://dx.doi.org/10.1016/j.ijid.2016.02.690]

[166] Verghese S. Post traumatic fungal keratitis caused by Acremonium recifei. Indian J Pathol Microbiol 2010; 53(3): 587-8. [http://dx.doi.org/10.4103/0377-4929.68263] [PMID: 20699545]

[167] Bharathi MJ, Ramakrishnan R, Meenakshi R, Shivakumar C, Raj DL. Analysis of the risk factors predisposing to fungal, bacterial \& Acanthamoeba keratitis in south India. Indian J Med Res 2009; 130(6): 749-57.

[PMID: 20090138]

[168] Reddy M, Venugopal R, Prakash PY, Kamath YS. Corneal ulcer due to a rare coelomycetes fungus Chaetomium strumarium: Case report and global review of Chaetomium keratomycosis. Indian J Ophthalmol 2017; 65(9): 871-4.

[http://dx.doi.org/10.4103/ijo.IJO 254 17] [PMID: 28905835]

[169] Bagyalakshmi R, Therese KL, Prasanna S, Madhavan HN. Newer emerging pathogens of ocular non-sporulating molds (NSM) identified by polymerase chain reaction (PCR)-based DNA sequencing technique targeting internal transcribed spacer (ITS) region. Curr Eye Res 2008; 33(2): 139-47.

[http://dx.doi.org/10.1080/02713680701864780] [PMID: 18293184]

[170] Sengupta J, Saha S, Khetan A, Ganguly A, Banerjee D. Candida fermentati: A rare yeast involved in fungal keratitis. Eye Contact Lens 2013; 39(4): e15-8.

[http://dx.doi.org/10.1097/ICL.0b013e318255121f] [PMID: 22669006]

[171] Qiu WY, Yao YF, Zhu YF, et al. Fungal spectrum identified by a new slide culture and in vitro drug susceptibility using Etest in fungal keratitis. Curr Eye Res 2005; 30(12): 1113-20. [http://dx.doi.org/10.1080/02713680500423671] [PMID: 16354625]

[172] Das S, Sharma S, Mahapatra S, Sahu SK. Fusarium keratitis at a tertiary eye care centre in India. Int Ophthalmol 2015; 35(3): 387-93. [http://dx.doi.org/10.1007/s10792-014-9961-5] [PMID: 24929671]

[173] He D, Hao J, Zhang B, et al. Pathogenic spectrum of fungal keratitis and specific identification of Fusarium solani. Invest Ophthalmol Vis Sci 2011; 52(5): 2804-8.

[http://dx.doi.org/10.1167/iovs.10-5977] [PMID: 21273551]

[174] Wong TY, Ng TP, Fong KS, Tan DT. Risk factors and clinical outcomes between fungal and bacterial keratitis: A comparative study. CLAO J 1997; 23(4): 275-81. [PMID: 9348453]

[175] Thomas PA, Kaliamurthy J. Mycotic keratitis: Epidemiology, diagnosis and management. Clin Microbiol Infect 2013; 19(3): 210-20. [http://dx.doi.org/10.1111/1469-0691.12126] [PMID: 23398543]

[176] Prajna NV, Krishnan T, Mascarenhas J, et al. The mycotic ulcer treatment trial: A randomized trial comparing natamycin vs voriconazole. JAMA Ophthalmol 2013; 131(4): 422-9.

[http://dx.doi.org/10.1001/jamaophthalmol.2013.1497] [PMID: 23710492]

[177] Prajna NV, Krishnan T, Rajaraman R, et al. Effect of oral voriconazole on fungal keratitis in the mycotic ulcer treatment trial ii [mutt ii]: A randomized clinical trial. JAMA Ophthalmol 2016; 134(12): $1365-72$.

[http://dx.doi.org/10.1001/jamaophthalmol.2016.4096] [PMID: 27787540]
[178] Anutarapongpan O. OBT. update on management of fungal keratitis. Clin Microbiol 2014; 3(5): 168.

[http://dx.doi.org/10.4172/2327-5073.1000168]

[179] FlorCruz NV, Evans JR. Medical interventions for fungal keratitis. Cochrane Database Syst Rev 2015; (4): CD004241 [4].

[PMID: 25855311]

[180] Arcieri ES, Rocha A, Mendonça CN, et al. Infectious keratitis secondary to Histoplasma capsulatum: The first case reports in humans. Braz J Infect Dis 2007; 11(6): 595-7.

[http://dx.doi.org/10.1590/S1413-86702007000600013] [PMID: 18327473]

[181] Jung SW, Kwon YA, Lee MK, Song SW. Epidermophyton fungal keratitis following laser-assisted subepithelial keratectomy. J Cataract Refract Surg 2009; 35(12): 2157-60.

[http://dx.doi.org/10.1016/j.jcrs.2009.06.035] [PMID: 19969224]

[182] Monden Y, Yamamoto S, Yamakawa R, et al. First case of fungal keratitis caused by Pestalotiopsis clavispora. Clin Ophthalmol 2013; 7 : 2261-4.

[http://dx.doi.org/10.2147/OPTH.S48732] [PMID: 24348013]

[183] Mravicić I, Dekaris I, Gabrić N, Romac I, Glavota V, Sviben M. Trichophyton Spp. fungal keratitis in 22 years old female contact lenses wearer. Coll Antropol 2010; 34(Suppl. 2): 271-4 [PMID: 21302731]

[184] Cheikhrouhou F, Makni F, Neji S, et al. Epidemiological profile of fungal keratitis in Sfax (Tunisia). J Mycol Med 2014; 24(4): 308-12. [Tunisia].

[http://dx.doi.org/10.1016/j.mycmed.2014.06.047] [PMID: 25442924]

[185] Mohammad A, Al-Rajhi A, Wagoner MD. Trichophyton fungal keratitis. Cornea 2006; 25(1): 118-22.

[http://dx.doi.org/10.1097/01.ico.0000164834.77291.51] [PMID: 16331054]

[186] Wang L, Sun S, Jing Y, Han L, Zhang H, Yue J. Spectrum of fungal keratitis in central China. Clin Exp Ophthalmol 2009; 37(8): 763-71. [http://dx.doi.org/10.1111/j.1442-9071.2009.02155.x] [PMID: 19878220]

[187] Jastaneiah SS, Al-Rajhi AA, Abbott D. Ocular mycosis at a referral center in Saudi Arabia: A 20-year study. Saudi J Ophthalmol 2011; 25(3): 231-8

[http://dx.doi.org/10.1016/j.sjopt.2011.04.004] [PMID: 23960930]

[188] Ruban VV, Kaliamurthy J, Dineshkumar M, Jesudasan CA, Geraldine P, Thomas PA. Keratitis due to the wood saprobic ascomycete, Auerswaldia lignicola (Family Botryosphaeriaceae), in a carpenter in India. Mycopathologia 2013; 176(5-6): 463-6.

[http://dx.doi.org/10.1007/s11046-013-9713-5] [PMID: 24158617]

[189] Kumar A, Pandya S, Kavathia G, Antala S, Madan M, Javdekar T. Microbial keratitis in Gujarat, Western India: findings from 200 cases. Pan Afr Med J 2011; 10: 48. [PMID: 22384294]

[190] Vaddavalli PK, Garg P, Sharma S, Sangwan VS, Rao GN, Thomas R. Role of confocal microscopy in the diagnosis of fungal and acanthamoeba keratitis. Ophthalmology 2011; 118(1): 29-35. [http://dx.doi.org/10.1016/j.ophtha.2010.05.018] [PMID: 20801515]

[191] Ghosh AK, Gupta A, Rudramurthy SM, Paul S, Hallur VK, Chakrabarti A. Fungal keratitis in north india: Spectrum of agents, risk factors and treatment. Mycopathologia 2016; 181(11-12): 843-50. [http://dx.doi.org/10.1007/s11046-016-0042-3] [PMID: 27473202]

[192] Lamarca J, Vilaplana F, Nadal J, García-Barberán I, Barraquer RI. Treatment resistant fungal keratitis caused by Colletotrichum gloeosporioides. Arch Soc Esp Oftalmol 2016; 91(2): 97-101. [http://dx.doi.org/10.1016/j.oftal.2015.07.008] [PMID: 26601974]

[193] He D, Hao J, Gao S, et al. Etiological Analysis of Fungal Keratitis and Rapid Identification of Predominant Fungal Pathogens. Mycopathologia 2016; 181(1-2): 75-82.

[http://dx.doi.org/10.1007/s11046-015-9950-x] [PMID: 26446032]

[194] Ritterband DC, Shah M, Seedor JA. Colletotrichum graminicola: A new corneal pathogen. Cornea 1997; 16(3): 362-4.

[http://dx.doi.org/10.1097/00003226-199705000-00019] [PMID: 9143813]

[195] Tabatabaei SA, Tabatabaei M, Soleimani M, Tafti ZF. Fungal keratitis caused by rare organisms. J Curr Ophthalmol 2017; 30(1): 91-6. [http://dx.doi.org/10.1016/j.joco.2017.08.004] [PMID: 29564417]

[196] Leck AK, Thomas PA, Hagan M, et al. Aetiology of suppurative corneal ulcers in Ghana and south India, and epidemiology of fungal keratitis. Br J Ophthalmol 2002; 86(11): 1211-5. [http://dx.doi.org/10.1136/bjo.86.11.1211] [PMID: 12386069]

[197] Chander J, Sharma A. Prevalence of fungal corneal ulcers in northern India. Infection 1994; 22(3): 207-9. 
[http://dx.doi.org/10.1007/BF01716706] [PMID: 7927819]

[198] Reinprayoon UPN, Kasetsuwan N, Plongla R, Mendoza L, Chindamporn A. Lagenidiumsp. ocular infection mimicking ocular pythiosis. Microbiol 2013; 13(51): 2778-80.

[199] Gajjar DU, Pal AK, Ghodadra BK, Vasavada AR. Microscopic evaluation, molecular identification, antifungal susceptibility, and clinical outcomes in fusarium, Aspergillus and, dematiaceous keratitis. BioMed Res Int 2013; 2013605308

[http://dx.doi.org/10.1155/2013/605308] [PMID: 24260740]

[200] Gajjar DU, Pal AK, Santos JM, Ghodadra BK, Vasavada AR. Severe pigmented keratitis caused by Cladorrhinum bulbillosum. Indian $\mathbf{J}$ Med Microbiol 2011; 29(4): 434-7.

[http://dx.doi.org/10.4103/0255-0857.90191] [PMID: 22120812]

[201] Tananuvat N, Salakthuantee K, Vanittanakom N, Pongpom M, Ausayakhun S. Prospective comparison between conventional microbial work-up vs PCR in the diagnosis of fungal keratitis. Eye (Lond) 2012; 26(10): 1337-43.

[http://dx.doi.org/10.1038/eye.2012.162] [PMID: 22878442]

[202] Iyer SA, Tuli SS, Wagoner RC. Fungal keratitis: emerging trends and treatment outcomes. Eye Contact Lens 2006; 32(6): 267-71.

[http://dx.doi.org/10.1097/01.icl.0000249595.27520.2e] [PMID: 17099386]

[203] Calvillo-Medina RP, Martínez-Neria M, Mena-Portales J, et al. Identification and biofilm development by a new fungal keratitis aetiologic agent. Mycoses 2019; 62(1): 62-72.

[http://dx.doi.org/10.1111/myc.12849] [PMID: 30187586]

[204] An N, Liu XN, Wang YN, et al. The pathogenic spectrum of fungal keratitis in northwestern China. Int J Ophthalmol 2016; 9(12): 1846-8. [PMID: 28003991]

[205] Theoulakis P, Goldblum D, Zimmerli S, Muehlethaler K, Frueh BE. Keratitis resulting from Thielavia subthermophila Mouchacca. Cornea 2009; 28(9): 1067-9.

[http://dx.doi.org/10.1097/ICO.0b013e31819717f4] [PMID: 19724200]

[206] Poria VC, Bharad VR, Dongre DS, Kulkarni MV. Study of mycotic keratitis. Indian J Ophthalmol 1985; 33(4): 229-31. [PMID: 3842831]

[207] Sharma S, Kunimoto DY, Gopinathan U, Athmanathan S, Garg P, Rao GN. Evaluation of corneal scraping smear examination methods in the diagnosis of bacterial and fungal keratitis: a survey of eight years of laboratory experience. Cornea 2002; 21(7): 643-7.

[http://dx.doi.org/10.1097/00003226-200210000-00002] [PMID: 12352078]

[208] Premamalini T, Ambujavalli BT, Vijayakumar R, Rajyoganandh SV, Kalpana S, Kindo AJ. Fungal keratitis caused by Macrophomina phaseolina - A case report. Med Mycol Case Rep 2012; 1(1): 123-6. [http://dx.doi.org/10.1016/j.mmcr.2012.10.007] [PMID: 24371757]

[209] Kindo AJ, Anita S, Kalpana S. Nattrassia mangiferae causing fungal keratitis. Indian J Med Microbiol 2010; 28(2): 178-81.

[http://dx.doi.org/10.4103/0255-0857.62504] [PMID: 20404473]

[210] Vanzzini Zago V, Manzano-Gayosso P, Hernández-Hernández F, Méndez-Tovar LJ, Gómez-Leal A, López Martínez R. Mycotic keratitis in an eye care hospital in Mexico City. Rev Iberoam Micol 2010; 27(2): 57-61. [Spanish].

[http://dx.doi.org/10.1016/j.riam.2009.09.003] [PMID: 20346302]

[211] Polack FMKH, Kaufman HE, Newmark E. Keratomycosis. Medical and surgical treatment. Arch Ophthalmol 1971; 85(4): 410-6. [http://dx.doi.org/10.1001/archopht.1971.00990050412003] [PMID: 4929071]

[212] Vislisel JM, Goins KM, Wagoner MD, et al. Incidence and outcomes of positive donor corneoscleral rim fungal cultures after keratoplasty. Ophthalmology 2017; 124(1): 36-42.

[http://dx.doi.org/10.1016/j.ophtha.2016.09.017] [PMID: 27817919]

[213] Suzuki T, Hori N, Miyake T, Hori Y, Mochizuki K. Keratitis caused by a rare fungus, Malassezia restricta. Jpn J Ophthalmol 2007; 51(4): 292-4.

[http://dx.doi.org/10.1007/s10384-007-0447-0] [PMID: 17660990]

[214] Balne PK, Nalamada S, Kodiganti M, Taneja M. Fungal keratitis caused by Chaetomium atrobrunneum. Cornea 2012; 31(1): 94-5. [http://dx.doi.org/10.1097/ICO.0b013e31821eeaed] [PMID: 22045390]

[215] Galarreta DJTS, Tuft SJ, Ramsay A, Dart JK. Fungal keratitis in London: Microbiological and clinical evaluation. Cornea 2007; 26(9): 1082-6.

[http://dx.doi.org/10.1097/ICO.0b013e318142bff3] [PMID: 17893539]

[216] Palanisamy M, Venkatapathy N, Rajendran V, Shobana CS. Keratomycosis caused by graphium eumorphum. J Clin Diagn Res
2015; 9(4): DD03-4 [PMID: 26023553]

[217] Eghrari AO, Gibas C, Watkins T, et al. First human case of fungal keratitis caused by a putatively novel species of lophotrichus. J Clin Microbiol 2015; 53(9): 3063-7.

[http://dx.doi.org/10.1128/JCM.00471-15] [PMID: 26109445]

[218] Wilde C, Messina M, Moshiri T, Snape SE, Maharajan S. Interface Scopulariopsis gracilis fungal keratitis following Descemet's stripping automated endothelial keratoplasty [DSAEK] with a contaminated graft. Int Ophthalmol 2017; 1-7. [PMID: 28900769]

[219] Srinivasan M, Gonzales CA, George C, et al. Epidemiology and aetiological diagnosis of corneal ulceration in Madurai, south India. $\mathrm{Br}$ J Ophthalmol 1997; 81(11): 965-71.

[http://dx.doi.org/10.1136/bjo.81.11.965] [PMID: 9505820]

[220] Chen HC, Tan HY, Hsiao CH, Huang SC, Lin KK, Ma DH. Amniotic membrane transplantation for persistent corneal ulcers and perforations in acute fungal keratitis. Cornea 2006; 25(5): 564-72. [http://dx.doi.org/10.1097/01.ico.0000227885.19124.6f] [PMID 16783145]

[221] Jhanji V, Yohendran J, Constantinou M, Sheorey H, Vajpayee RB. Scedosporium scleritis or keratitis or both: Case series. Eye Contact Lens 2009; 35(6): 312-5.

[http://dx.doi.org/10.1097/ICL.0b013e3181be722e] [PMID: 19816184]

[222] Ritterband DC, Seedor JA, Shah MK, Koplin RS, McCormick SA Fungal keratitis at the new york eye and ear infirmary. Cornea 2006; 25(3): 264-7.

[http://dx.doi.org/10.1097/01.ico.0000177423.77648.8d] [PMID: 16633023]

[223] Derhy D, Sauer A, Sabou M, et al. Surgical treatment of Metarhizium anisopliae sclerokeratitis and endophthalmitis. Indian J Ophthalmol 2017; 65(6): 523-6.

[http://dx.doi.org/10.4103/ijo.IJO 461 16] [PMID: 28643721]

[224] Jani BR, Rinaldi MG, Reinhart WJ. An unusual case of fungal keratitis: Metarrhizium anisopliae. Cornea 2001; 20(7): 765-8. [http://dx.doi.org/10.1097/00003226-200110000-00020] [PMID: 11588434]

[225] Shah CV, Jones DB, Holz ER. Microsphaeropsis olivacea keratitis and consecutive endophthalmitis. Am J Ophthalmol 2001; 131(1): 142-3. [http://dx.doi.org/10.1016/S0002-9394(00)00715-7] [PMID: 11162997]

[226] Gupta A, Capoor MR, Gupta S, Kochhar S, Tomer A, Gupta V. Clinico-demographical profile of keratomycosis in Delhi, North India. Indian J Med Microbiol 2014; 32(3): 310-4.

[http://dx.doi.org/10.4103/0255-0857.136582] [PMID: 25008827]

[227] Kotigadde SBM. Jyothirlatha, Kumar A, Srinivasa R, Shivananda PG. Mycotic keratitis: A study in coastal karnataka. Indian J Ophthalmol 1992; 33(4): 229-31.

[228] Xie L, Zhong W, Shi W, Sun S. Spectrum of fungal keratitis in north China. Ophthalmology 2006; 113(11): 1943-8. [http://dx.doi.org/10.1016/j.ophtha.2006.05.035] [PMID: 16935335]

[229] Figueira L, Pinheiro D, Moreira R, et al. Beauveria bassiana keratitis in bullous keratopathy: Antifungal sensitivity testing and management. Eur J Ophthalmol 2012; 22(5): 814-8.

[http://dx.doi.org/10.5301/ejo.5000152] [PMID: 22467587]

[230] Pariseau B, Nehls S, Ogawa GS, Sutton DA, Wickes BL, Romanelli AM. Beauveria keratitis and biopesticides: Case histories and a random amplification of polymorphic DNA comparison. Cornea 2010; 29(2): 152-8.

[http://dx.doi.org/10.1097/ICO.0b013e3181ae2575]

[PMID: 20023588]

[231] Panda A, Sharma N, Das G, Kumar N, Satpathy G. Mycotic keratitis in children: Epidemiologic and microbiologic evaluation. Cornea 1997; 16(3): 295-9.

[http://dx.doi.org/10.1097/00003226-199705000-00007] [PMID: 9143801]

[232] Vyas N, Al-Hashimi S, Munir W. Microcyclosporella mali: A novel fungal keratitis in a post-penetrating keratoplasty patient. BMJ Case Rep 2015; 2015: 27.

[http://dx.doi.org/10.1136/bcr-2014-207416] [PMID: 25725025]

[233] Bennett HG, Hay J, Kirkness CM, Seal DV, Devonshire P. Antimicrobial management of presumed microbial keratitis: Guidelines for treatment of central and peripheral ulcers. $\mathrm{Br} \mathrm{J}$ Ophthalmol 1998; 82(2): 137-45.

[http://dx.doi.org/10.1136/bjo.82.2.137] [PMID: 9613378]

[234] Naiker S, Odhav B. Mycotic keratitis: profile of Fusarium species and 
their mycotoxins. Mycoses 2004; 47(1-2): 50-6. [50-56] [http://dx.doi.org/10.1046/j.0933-7407.2003.00936.x]

[PMID: 14998400]

[235] Esposto MC, Prigitano A, Tortorano AM. Fusarium musae as cause of superficial and deep-seated human infections. J Mycol Med 2016; 26(4): 403-5

[http://dx.doi.org/10.1016/j.mycmed.2016.02.021] [PMID: 27091579]

[236] Jin $\mathrm{X}$, Zhao $\mathrm{Y}$, Zhang $\mathrm{F}$, et al. Neutrophil extracellular traps involvement in corneal fungal infection. Mol Vis 2016; 22: 944-52. [PMID: 27559290]

[237] Chodosh J, Miller D, Tu EY, Culbertson WW. Tobramycin-responsive Fusarium oxysporum keratitis. Can J Ophthalmol 2000; 35(1): 29-30. [http://dx.doi.org/10.1016/S0008-4182(00)80107-6] [PMID: 10711382]

[238] Xie L, Zhai H, Zhao J, Sun S, Shi W, Dong X. Antifungal susceptibility for common pathogens of fungal keratitis in Shandong Province, China. Am J Ophthalmol 2008; 146(2): 260-5. [http://dx.doi.org/10.1016/j.ajo.2008.04.019] [PMID: 18547535]

[239] Tupaki-Sreepurna A, Al-Hatmi AMS, Kindo AJ, Sundaram M, de Hoog GS. Multidrug-resistant fusarium in keratitis: A clinicomycological study of keratitis infections in Chennai, India. Mycoses 2017; 60(4): 230-3.

[http://dx.doi.org/10.1111/myc.12578] [PMID: 27766684]

[240] Gharamah AA, Moharram AM, Ismail MA, Al-Hussaini AK. Bacterial and fungal keratitis in Upper Egypt: In vitro screening of enzymes, toxins and antifungal activity. Indian J Ophthalmol 2014; 62(2): 196-203.

[http://dx.doi.org/10.4103/0301-4738.116463] [PMID: 24008795]

[241] Guarro J, Rubio C, Gené J, et al. Case of keratitis caused by an uncommon Fusarium species. J Clin Microbiol 2003; 41(12): 5823-6. [http://dx.doi.org/10.1128/JCM.41.12.5823-5826.2003] [PMID: 14662993]

[242] Hassan AS, Al-Hatmi AM, Shobana CS, et al. Antifungal susceptibility and phylogeny of opportunistic members of the genus fusarium causing human keratomycosis in south india. Med Mycol 2016; 54(3): 287-94.

[http://dx.doi.org/10.1093/mmy/myv105] [PMID: 26705832]

[243] do Carmo A, Costa E, Marques M, Quadrado MJ, Tomé R. Fusarium dimerum Species Complex (Fusarium penzigii) Keratitis After Corneal Trauma. Mycopathologia 2016; 181(11-12): 879-84. [Fusarium penzigii].

[http://dx.doi.org/10.1007/s11046-016-0060-1] [PMID: 27631818]

[244] Homa M, Shobana CS, Singh YRB, et al. Fusarium keratitis in South India: causative agents, their antifungal susceptibilities and a rapid identification method for the Fusarium solani species complex. Mycoses 2013; 56(5): 501-11.

[http://dx.doi.org/10.1111/myc.12062] [PMID: 23437826]

[245] Al-Hatmi AMS, Bonifaz A, de Hoog GS, et al. Keratitis by Fusarium temperatum, a novel opportunist. BMC Infect Dis 2014; 14: 588. [1]. [http://dx.doi.org/10.1186/s12879-014-0588-y] [PMID: 25388601]

[246] Kunimoto DY, Sharma S, Garg P, Gopinathan U, Miller D, Rao GN. Corneal ulceration in the elderly in Hyderabad, south India. Br J Ophthalmol 2000; 84(1): 54-9.

[http://dx.doi.org/10.1136/bjo.84.1.54] [PMID: 10611100 ]

[247] Ibrahim MM, Vanini R, Ibrahim FM, et al. Epidemiologic aspects and clinical outcome of fungal keratitis in southeastern Brazil. Eur $\mathbf{J}$ Ophthalmol 2009; 19(3): 355-61

[http://dx.doi.org/10.1177/112067210901900305] [PMID: 19396778]

[248] Manikandan P, Vismer HF, Kredics L, et al. Corneal ulcer due to Neocosmospora vasinfecta in an immunocompetent patient. Med Mycol 2008; 46(3): 279-84.

[http://dx.doi.org/10.1080/13693780701625149] [PMID: 17885942]

[249] Dunlop AA, Wright ED, Howlader SA, et al. Suppurative corneal ulceration in Bangladesh. A study of 142 cases examining the microbiological diagnosis, clinical and epidemiological features of bacterial and fungal keratitis. Aust N Z J Ophthalmol 1994; 22(2): 105-10.

[http://dx.doi.org/10.1111/j.1442-9071.1994.tb00775.x] [PMID: 7917262]

[250] Guarro J, Höfling-Lima AL, Gené J, et al. Corneal ulcer caused by the new fungal species Sarcopodium oculorum. J Clin Microbiol 2002; 40(8): 3071-5

[http://dx.doi.org/10.1128/JCM.40.8.3071-3075.2002] [PMID: 12149384]

[251] Yamada H, Takahashi N, Hori N, et al. Rare case of fungal keratitis caused by Corynespora cassiicola. J Infect Chemother 2013; 19(6): 1167-9. [http://dx.doi.org/10.1007/s10156-013-0579-8] [PMID: 23494266]

[252] Huang YHLI, Lin IH, Chang TC, Tseng SH. Early diagnosis and successful treatment of Cryptococcus albidus keratitis: A case report and literature review. Medicine (Baltimore) 2015; 94(19)e885 [http://dx.doi.org/10.1097/MD.0000000000000885]

[PMID: 25984681]

[253] Ting DSJBG, Koerner R, Irion LD, Johnson E, Morgan SJ, Ghosh S. Polymicrobial keratitis with cryptococcus curvatus, candida parapsilosis, and stenotrophomonas maltophilia after penetrating keratoplasty: A rare case report with literature review. Eye \& Contact Lens: Science \&. Clin Pract 2019; 45(2): 5-10.

[254] Bhandary SVVH, VijayaPai H, Rao LG, Yegneswaran PP. Cunninghamella spinosum fungal corneal ulcer- first case report. Indian J Ophthalmol 2014; 62(3): 375-6.

[http://dx.doi.org/10.4103/0301-4738.130436] [PMID: 24722275]

[255] Juyal D, Pal S, Sharma M, Negi V, Adekhandi S, Tyagi M. Keratomycosis due to Purpureocillium lilacinum: A case report from Sub-Himalayan region of Uttarakhand. Indian J Pathol Microbiol 2018; 61(4): 607-9.

[http://dx.doi.org/10.4103/IJPM.IJPM 404 17] [PMID: 30303164]

[256] Morrison AS, Lockhart SR, Bromley JG, Kim JY, Burd EM. An environmental Sporothrix as a cause of corneal ulcer. Med Mycol Case Rep 2013; 2(1): 88-90.

[http://dx.doi.org/10.1016/j.mmcr.2013.03.002] [PMID: 24432225]

[257] Mascarenhas J, Lalitha P, Prajna NV, et al. Acanthamoeba, fungal, and bacterial keratitis: A comparison of risk factors and clinical features. Am J Ophthalmol 2014; 157(1): 56-62.

[http://dx.doi.org/10.1016/j.ajo.2013.08.032] [PMID: 24200232]

[258] Thomas PAKT, Kuriakose T. Keratitis due to Arthrobotrys oligospora Fres. 1850. J Med Vet Mycol 1990; 28(1): 47-50. [http://dx.doi.org/10.1080/02681219080000061] [PMID: 2362231]

[259] Ranjith K, Sontam B, Sharma S, et al. Candida species from eye infections: Drug susceptibility, virulence factors, and molecular characterization. Invest Ophthalmol Vis Sci 2017; 58(10): 4201-9.

[http://dx.doi.org/10.1167/iovs.17-22003] [PMID: 28837732]

[260] Ahmed SA, Hofmüller W, Seibold M, et al. Tintelnotia, a new genus in Phaeosphaeriaceae harbouring agents of cornea and nail infections in humans. Mycoses 2017; 60(4): 244-53.

[http://dx.doi.org/10.1111/myc.12588] [PMID: 27910191]

[261] Bhartiya P, Daniell M, Constantinou M, Islam FM, Taylor HR. Fungal keratitis in Melbourne. Clin Exp Ophthalmol 2007; 35(2): 124-30. [PMID: 17362452]

[262] Mandell KJ, Colby KA. Penetrating keratoplasty for invasive fungal keratitis resulting from a thorn injury involving Phomopsis species. Cornea 2009; 28(10): 1167-9.

[http://dx.doi.org/10.1097/ICO.0b013e31819839e6]

[PMID: 19770729]

[263] Gajjar DUPA, Pal AK, Parmar TJ, et al. Fungal scleral keratitis caused by Phomopsis phoenicicola. J Clin Microbiol 2011; 49(6): 2365-8. [http://dx.doi.org/10.1128/JCM.02449-10] [PMID: 21450952]

[264] Khanal B, Kaini KR, Deb M, Badhu B, Thakur SK. Microbial keratitis in eastern Nepal. Trop Doct 2001; 31(3): 168-9.

[http://dx.doi.org/10.1177/004947550103100319] [PMID: 11444343]

[265] Kamada R, Monden Y, Uehara K, Yamakawa R, Nishimura K. Rare case of fungal keratitis caused by Plectosporium tabacinum. Clin Ophthalmol 2012; 6(1): 1623-7.

[http://dx.doi.org/10.2147/OPTH.S36318] [PMID: 23055688]

[266] Dudeja LLJ, Karpagam R, Venkatesh P, Lalitha P. Fungal keratitis caused by epicoccum sorghi - A case report. Delhi J Ophthalmol 2016; 27: 121-3.

[http://dx.doi.org/10.7869/djo.223]

[267] da Cunha KC, Sutton DA, Fothergill AW, et al. In vitro antifungal susceptibility and molecular identity of 99 clinical isolates of the opportunistic fungal genus Curvularia. Diagn Microbiol Infect Dis 2013; 76(2): 168-74.

[http://dx.doi.org/10.1016/j.diagmicrobio.2013.02.034]

[PMID: 23558007]

[268] Guarro J, Akiti T, Horta RA, et al. Mycotic keratitis due to Curvularia senegalensis and in vitro antifungal susceptibilities of Curvularia spp. J Clin Microbiol 1999; 37(12): 4170-3. [PMID: 10565956]

[269] Forster RK, Rebell G, Wilson LA. Dematiaceous fungal keratitis. Clinical isolates and management. Br J Ophthalmol 1975; 59(7): 372-6.

[http://dx.doi.org/10.1136/bjo.59.7.372] [PMID: 1081406]

[270] Kibret T, Bitew A. Fungal keratitis in patients with corneal ulcer attending Minilik II Memorial Hospital, Addis Ababa, Ethiopia. BMC 
Ophthalmol 2016; 16(1): 148

[http://dx.doi.org/10.1186/s12886-016-0330-1] [PMID: 27576913]

[271] Ferrer C, Montero J, Alió JL, Abad JL, Ruiz-Moreno JM, Colom F. Rapid molecular diagnosis of posttraumatic keratitis and endophthalmitis caused by Alternaria infectoria. J Clin Microbiol 2003; 41(7): 3358-60.

[http://dx.doi.org/10.1128/JCM.41.7.3358-3360.2003] [PMID: 12843093]

[272] Hua Gao PS, Jose J. Echegaray. Yanni Jia, Suxia Li, Man Du, Victor L. Perez, and weiyun shi. big bubble deep anterior lamellar keratoplasty for management of deep fungal keratitis. J Ophthalmol 2014.

[273] Chowdhary A, Singh K. Spectrum of fungal keratitis in North India. Cornea 2005; 24(1): 8-15

[http://dx.doi.org/10.1097/01.ico.0000126435.25751.20] [PMID: 15604861]

[274] Wang L, Al-Hatmi AMS, Lai X, et al. Bipolaris oryzae, a novel fungal opportunist causing keratitis. Diagn Microbiol Infect Dis 2016; 85(1): $61-5$.

[http://dx.doi.org/10.1016/j.diagmicrobio.2015.11.020] [PMID: 26976720]

[275] Ormerod LD, Smith RE. Contact lens-associated microbial keratitis. Arch Ophthalmol 1986; 104(1): 79-83.

[http://dx.doi.org/10.1001/archopht.1986.01050130089027] [PMID: 3942549]

[276] Sengupta S, Thiruvengadakrishnan K, Ravindran RD, Vaitilingam MC. Changing referral patterns of infectious corneal ulcers to a tertiary care facility in south India - 7-year analysis. Ophthalmic Epidemiol 2012; 19(5): 297-301.

[http://dx.doi.org/10.3109/09286586.2012.690492] [PMID: 22897620]

[277] Ferrer C, Pérez-Santonja JJ, Rodríguez AE, et al. New pyrenochaeta species causing keratitis. J Clin Microbiol 2009; 47(5): 1596-8. [http://dx.doi.org/10.1128/JCM.01912-08] [PMID: 19297598]

[278] Verkley GJGJ, Gené J, Guarro J, et al. Pyrenochaeta keratinophila sp. nov., isolated from an ocular infection in Spain. Rev Iberoam Micol 2010; 27(1): 22-4.

[http://dx.doi.org/10.1016/j.riam.2009.09.001] [PMID: 19955009]

[279] Hotta F, Eguchi H, Nishimura K, et al. A super-infection in the cornea caused by Stemphylium, Acremonium, and $\alpha$-Streptococcus. Ann Clin Microbiol Antimicrob 2017; 16(1): 11. [1]. [no pagination]. [11]. [http://dx.doi.org/10.1186/s12941-017-0187-z] [PMID: 28279173]

[280] Badenoch PRHC, Halliday CL, Ellis DH, Billing KJ, Mills RA. Ulocladium atrum keratitis. J Clin Microbiol 2006; 44(3): 1190-3. [1190-3].

[http://dx.doi.org/10.1128/JCM.44.3.1190-1193.2006] [PMID: 16517929]

[281] Al-Shakarchi F. Initial therapy for suppurative microbial keratitis in Iraq. Br J Ophthalmol 2007; 91(12): 1583-7. [http://dx.doi.org/10.1136/bjo.2007.123208] [PMID: 17596332]

[282] Shah A, Mauger T. Magnusiomyces capitatus: a new and emerging pathogen linked to keratomycosis. Digit J Ophthalmol 2017; 23(3): $75-7$

[http://dx.doi.org/10.5693/djo.02.2017.04.001] [PMID: 29162991]

[283] Rautaraya B, Sharma S, Kar S, Das S, Sahu SK. Diagnosis and treatment outcome of mycotic keratitis at a tertiary eye care center in eastern India. BMC Ophthalmol 2011; 11:39. [http://dx.doi.org/10.1186/1471-2415-11-39] [PMID: 22188671]

[284] Biser SA, Perry HD, Donnenfeld ED, Doshi SJ, Chaturvedi V. Arthrographis keratitis mimicking acanthamoeba keratitis. Cornea 2004; 23(3): 314-7.

[http://dx.doi.org/10.1097/00003226-200404000-00018] [PMID: 15084869]

[285] Al-Falki YH, Alshehri MA, Joseph MRP, Hamid ME. Fungal keratitis caused by a rare ocular pathogen, Gjaerumia minor: A case report. Saudi J Ophthalmol 2017 [PMID: 29942188]

[286] Wessel JM, Bachmann BO, Meiller R, Kruse FE. Fungal interface keratitis by Candida orthopsilosis following deep anterior lamellar keratoplasty. BMJ Case Rep 2013; 2013: 23.

[http://dx.doi.org/10.1136/bcr-2012-008361] [PMID: 23349184]

[287] Imwidthaya P. Mycotic keratitis in Thailand. J Med Vet Mycol 1995; 33(1): $81-2$.

[http://dx.doi.org/10.1080/02681219580000171] [PMID: 7650585]

[288] Reddy AK, Ashok R, Majety M, Chitta M, Narayen N. Fungal keratitis due to Schizophyllum commune: An emerging pathogenic fungus. Mycoses 2016; 59(12): 757-9.

[http://dx.doi.org/10.1111/myc.12527] [PMID: 27402206]
[289] Saeedi OJ, Iyer SA, Mohiuddin AZ, Hogan RN. Exophiala jeanselmei keratitis: Case report and review of literature. Eye Contact Lens 2013; 39(6): 410-2.

[http://dx.doi.org/10.1097/ICL.0b013e3182993901] [PMID: 24045832]

[290] Aggarwal S, Yamaguchi T, Dana R, Hamrah P. Exophiala phaeomuriformis Fungal Keratitis: Case Report and In Vivo Confocal Microscopy Findings. Eye Contact Lens 2017; 43(2): e4-6. [http://dx.doi.org/10.1097/ICL.0000000000000193]

[PMID: 26513718]

[291] Xie L, Zhai H, Shi W. Penetrating keratoplasty for corneal perforations in fungal keratitis. Cornea 2007; 26(2): 158-62. [http://dx.doi.org/10.1097/01.ico.0000248381.24519.0d] [PMID 17251805]

[292] Patel SR, Hammersmith KM, Rapuano CJ, Cohen EJ. Exophiala dermatitidis keratitis after laser in situ keratomileusis. J Cataract Refract Surg 2006; 32(4): 681-4.

[http://dx.doi.org/10.1016/j.jcrs.2006.01.040] [PMID: 16698496]

[293] Tilak R, Singh A, Maurya OPS, Chandra A, Tilak V, Gulati AK. Mycotic keratitis in India: A five-year retrospective study. J Infect Dev Ctries 2010; 4(3): 171-4

[http://dx.doi.org/10.3855/jidc.309] [PMID: 20351459]

[294] Khanal B, Deb M, Panda A, Sethi HS. Laboratory diagnosis in ulcerative keratitis. Ophthalmic Res 2005; 37(3): 123-7. [http://dx.doi.org/10.1159/000084273] [PMID: 15746569]

[295] Garg P, Gopinathan U, Choudhary K, Rao GN. Keratomycosis: Clinical and microbiologic experience with dematiaceous fungi. Ophthalmology 2000; 107(3): 574-80.

[http://dx.doi.org/10.1016/S0161-6420(99)00079-2]

[PMID: 10711898]

[296] Pushker N, Dada T, Sony P, Ray M, Agarwal T, Vajpayee RB. Microbial keratitis after laser in situ keratomileusis. J Refract Surg 2002; 18(3): 280-6.

[PMID: 12051385]

[297] Shigeyasu C, Yamada M, Nakamura N, Mizuno Y, Sato T, Yaguchi T. Keratomycosis caused by Aspergillus viridinutans: An Aspergillus fumigatus-resembling mold presenting distinct clinical and antifungal susceptibility patterns. Med Mycol 2012; 50(5): 525-8.

[http://dx.doi.org/10.3109/13693786.2012.658875] [PMID: 22329455]

[298] Dalmon C, Porco TC, Lietman TM, et al. The clinical differentiation of bacterial and fungal keratitis: A photographic survey. Invest Ophthalmol Vis Sci 2012; 53(4): 1787-91.

[http://dx.doi.org/10.1167/iovs.11-8478] [PMID: 22395880]

[299] Wang L, Wang L, Han L, Yin W. Study of pathogens of fungal keratitis and the sensitivity of pathogenic fungi to therapeutic agents with the disk diffusion method. Curr Eye Res 2015; 40(11): 1095-101. [http://dx.doi.org/10.3109/02713683.2015.1056802] [PMID: 26268399]

[300] Baranyi N, Kocsubé S, Szekeres A, et al. Keratitis caused by Aspergillus pseudotamarii. Med Mycol Case Rep 2013; 2(1): 91-4. [http://dx.doi.org/10.1016/j.mmcr.2013.04.002] [PMID: 24432226]

[301] Manikandan P, Varga J, Kocsubé S, et al. Mycotic keratitis due to Aspergillus nomius. J Clin Microbiol 2009; 47(10): 3382-5. [http://dx.doi.org/10.1128/JCM.01051-09] [PMID: 19710265]

[302] Manikandan P, Varga J, Kocsubé S, et al. Epidemiology of Aspergillus keratitis at a tertiary care eye hospital in South India and antifungal susceptibilities of the causative agents. Mycoses 2013; 56(1): 26-33.

[http://dx.doi.org/10.1111/j.1439-0507.2012.02194.x] [PMID: 22487304]

[303] Posteraro B, Mattei R, Trivella F, et al. Uncommon Neosartorya udagawae fungus as a causative agent of severe corneal infection. J Clin Microbiol 2011; 49(6): 2357-60.

[http://dx.doi.org/10.1128/JCM.00134-11] [PMID: 21450961]

[304] Hsieh H-MJ. Fungal keratitis caused by a new filamentous hyphomycete Sagenomella keratitidis. Bot Stud (Taipei, Taiwan) 2009; 50(3): 331-5.

[305] Gonawardena SA, Ranasinghe KP, Arseculeratne SN, Seimon CR, Ajello L. Survey of mycotic and bacterial keratitis in Sri Lanka. Mycopathologia 1994; 127(2): 77-81. [http://dx.doi.org/10.1007/BF01103062] [PMID: 7984216]

[306] Vyawahare CR, Misra RN, Gandham NR, Angadi KM, Paul R. Penicillium keratitis in an immunocompetent patient from Pune, Maharashtra, India. J Clin Diagn Res 2014; 8(7): DD01-2. [http://dx.doi.org/10.7860/JCDR/2014/7996.4647] [PMID: 25177564]

[307] Ghosh A, Basu S, Datta H, Chattopadhyay D. Evaluation of polymerase chain reaction-based ribosomal DNA sequencing 
technique for the diagnosis of mycotic keratitis. Am J Ophthalmol 2007; 144(3): 396-403.

[http://dx.doi.org/10.1016/j.ajo.2007.05.017] [PMID: 17631849]

[308] Bawazeer AM, Hodge WG. Rhodotorula infection in a corneal graft following penetrating keratoplasty. Can J Ophthalmol 2003; 38(3): $225-7$.

[http://dx.doi.org/10.1016/S0008-4182(03)80065-0] [PMID: 12733691]

[309] Ahn M, Yoon KC, Ryu SK, Cho NC, You IC. Clinical aspects and prognosis of mixed microbial (bacterial and fungal) keratitis. Cornea 2011; 30(4): 409-13.

[http://dx.doi.org/10.1097/ICO.0b013e3181f23704] [PMID: 21045645]

[310] Giovannini J, Lee R, Zhang SX, Jun AS, Bower KS. Rhodotorula keratitis: a rarely encountered ocular pathogen. Case Rep Ophthalmol 2014; 5(3): 302-10.

[http://dx.doi.org/10.1159/000365986] [PMID: 25408670]

[311] Saha S, Sengupta J, Chatterjee D, Banerjee D. Rhodotorula mucilaginosa Keratitis: A rare fungus from Eastern India. Indian $\mathrm{J}$ Ophthalmol 2014; 62(3): 341-4.

[http://dx.doi.org/10.4103/0301-4738.111133] [PMID: 23619486]

[312] Hagan M, Wright E, Newman M, Dolin P, Johnson G. Causes of suppurative keratitis in Ghana. Br J Ophthalmol 1995; 79(11): 1024-8. [http://dx.doi.org/10.1136/bjo.79.11.1024] [PMID: 8534648]

[313] Sane S, Sharma S, Konduri R, Fernandes M. Emerging corneal pathogens: First report of Pseudopestalotiopsis theae keratitis. Indian J Ophthalmol 2019; 67(1): 150-2.

[http://dx.doi.org/10.4103/ijo.IJO_791_18] [PMID: 30574929]

[314] Sun JP, Chen WL, Huang JY, Hou YC, Wang IJ, Hu FR. Microbial keratitis after penetrating keratoplasty. Am J Ophthalmol 2017; 178: $150-6$.

[http://dx.doi.org/10.1016/j.ajo.2017.03.022] [PMID: 28347669]

[315] Chew HF, Jungkind DL, Mah DY, et al. Post-traumatic fungal keratitis caused by Carpoligna sp. Cornea 2010; 29(4): 449-52

[http://dx.doi.org/10.1097/ICO.0b013e3181af3954]

[PMID: 20168220]

[316] Ramakrishnan T, Constantinou M, Jhanji V, Vajpayee RB. Factors affecting treatment outcomes with voriconazole in cases with fungal keratitis. Cornea 2013; 32(4): 445-9.

[http://dx.doi.org/10.1097/ICO.0b013e318254a41b] [PMID: 22580440]

[317] Punia RS, Kundu R, Chander J, Arya SK, Handa U, Mohan H. Spectrum of fungal keratitis: Clinicopathologic study of 44 cases. Int $\mathbf{J}$ Ophthalmol 2014; 7(1): 114-7. [PMID: 24634875]

[318] Kamoshita M, Matsumoto Y, Nishimura K, et al. Wickerhamomyces anomalus fungal keratitis responds to topical treatment with antifungal micafungin. J Infect Chemother 2015; 21(2): 141-3 [http://dx.doi.org/10.1016/j.jiac.2014.08.019] [PMID: 25239058]

[319] Tsatsos M, MacGregor C, Athanasiadis I, Moschos MM, Hossain P, Anderson D. Herpes simplex virus keratitis: An update of the pathogenesis and current treatment with oral and topical antiviral agents. Clin Exp Ophthalmol 2016; 44(9): 824-37. [http://dx.doi.org/10.1111/ceo.12785] [PMID: 27273328]

[320] Vadoothker S, Andrews L, Jeng BH, Levin MR. Management of Herpes Simplex Virus Keratitis in the Pediatric Population. Pediatr Infect Dis J 2018; 37(9): 949-51.

[http://dx.doi.org/10.1097/INF.0000000000002114] [PMID: 29794647]

[321] Bhatt UK, Abdul Karim MN, Prydal JI, Maharajan SV, Fares U. Oral antivirals for preventing recurrent herpes simplex keratitis in people with corneal grafts. Cochrane Database Syst Rev 2016; 11CD007824 [11].

[http://dx.doi.org/10.1002/14651858.CD007824.pub2] [PMID: 27902849]

[322] McDonald EM, Patel DV, McGhee CN. A prospective study of the clinical characteristics of patients with herpes simplex and varicella zoster keratitis, presenting to a New Zealand emergency eye clinic. Cornea 2015; 34(3): 279-84.

[http://dx.doi.org/10.1097/ICO.0000000000000338] [PMID: 25532996]

[323] Szeto SKHM, Chan TCYF, Wong RLMM, Ng ALKM, Li EYMF, Jhanji V. Prevalence of Ocular Manifestations and Visual Outcomes in Patients With Herpes Zoster Ophthalmicus. Cornea 2017; 36(3): 338-42.

[PMID: 27741018]

[324] Wilhelmus KR, Beck RW, Moke PS, et al. Acyclovir for the prevention of recurrent herpes simplex virus eye disease. N Engl J Med 1998; 339(5): 300-6.

[http://dx.doi.org/10.1056/NEJM199807303390503] [PMID: 9696640]

[325] White MCJ. Herpes Simples Virus Keratitis: A Treatment Guideline. American Academy of Ophthalmology 2014.

[326] Hoyle E, Erez JC, Kirk-Granger HR, Collins E, Tang JW. An adenovirus 4 outbreak amongst staff in a pediatric ward manifesting as keratoconjunctivitis-a possible failure of contact and aerosol infection control. Am J Infect Control 2016; 44(5): 602-4.

[http://dx.doi.org/10.1016/j.ajic.2015.11.032] [PMID: 26804304]

[327] Chodosh J, Miller D, Stroop WG, Pflugfelder SC. Adenovirus epithelial keratitis. Cornea 1995; 14(2): 167-74.

[http://dx.doi.org/10.1097/00003226-199503000-00010] [PMID: 7743800]

[328] Wilhelmus KRFR, Font RL, Lehmann RP, Cernoch PL. Cytomegalovirus keratitis in acquired immunodeficiency syndrome. Arch Ophthalmol 1996; 114(7): 869-72.

[http://dx.doi.org/10.1001/archopht.1996.01100140083016] [PMID: 8660174]

[329] Matoba AY, Wilhelmus KR, Jones DB. Epstein-Barr viral stromal keratitis. Ophthalmology 1986; 93(6): 746-51.

[http://dx.doi.org/10.1016/S0161-6420(86)33668-6] [PMID: 3737121]

[330] Rowe AM, Yun H, Hendricks RL. Exposure stress induces reversible corneal graft opacity in recipients with herpes simplex virus-1 infections. Invest Ophthalmol Vis Sci 2017; 58(1): 35-41. [http://dx.doi.org/10.1167/iovs.16-19673] [PMID: 28055100]

[331] Grillo AP, Fraunfelder FW. Keratitis in association with herpes zoster and varicella vaccines. Drugs Today (Barc) 2017; 53(7): 393-7. [http://dx.doi.org/10.1358/dot.2017.53.7.2667582] [PMID: 28837183]

[332] Pavlopoulos GP, Giannakos GI, Theodosiadis PG, Moschos MM, Iliakis EK, Theodosiadis GP. Rubeola keratitis: A photographic study of corneal lesions. Cornea 2008; 27(4): 411-6. [http://dx.doi.org/10.1097/ICO.0b013e31816313a2]

[PMID: 18434843]

[333] Onal S, Toker E. A rare ocular complication of mumps: Keratouveitis. Ocul Immunol Inflamm 2005; 13(5): 395-7.

[http://dx.doi.org/10.1080/09273940590950927] [PMID: 16419425]

[334] Kaye SB, Morton CE, Tong CY, O’Donnell NP. Echovirus keratoconjunctivitis. Am J Ophthalmol 1998; 125(2): 187-90.

[http://dx.doi.org/10.1016/S0002-9394(99)80090-7] [PMID: 9467445]

[335] Ruben FL, Lane JM, Lane M. Ocular vaccinia. An epidemiologic analysis of 348 cases. Arch Ophthalmol 1970; 84(1): 45-8.

[http://dx.doi.org/10.1001/archopht.1970.00990040047012] [PMID: 5423606]

[336] Pepose JS, Margolis TP, LaRussa P, Pavan-Langston D. Ocula complications of smallpox vaccination. Am J Ophthalmol 2003; 136(2): 343-52.

[http://dx.doi.org/10.1016/S0002-9394(03)00293-9] 12888060]

[337] Clarke DW, Niederkorn JY. The pathophysiology of acanthamoeba keratitis. Trends Parasitol 2006; 22(4): 175-80. [http://dx.doi.org/10.1016/j.pt.2006.02.004] [PMID: 16500148]

[338] Maycock NJRBMF, Jayaswal R. Update on acanthamoeba keratitis: Diagnosis, treatment, and outcomes. Cornea 2016; 35(5): 713-20. [http://dx.doi.org/10.1097/ICO.0000000000000804] [PMID: 26989955]

[339] McKelvie J, Alshiakhi M, Ziaei M, Patel DV, McGhee CN. The rising tide of Acanthamoeba keratitis in Auckland, New Zealand: A 7-year review of presentation, diagnosis and outcomes (2009-2016). Clin Exp Ophthalmol 2018; 46(6): 600-7. [2009-2016].

[http://dx.doi.org/10.1111/ceo.13166] [PMID: 29412494]

[340] Niederkorn JY, Alizadeh H, Leher H, McCulley JP. The pathogenesis of Acanthamoeba keratitis. Microbes Infect 1999; 1(6): 437-43. [http://dx.doi.org/10.1016/S1286-4579(99)80047-1] [PMID: 10602676]

[341] Tawfeek GM, Bishara SAH, Sarhan RM, ElShabrawi Taher E, ElSaady Khayyal A. Genotypic, physiological, and biochemical characterization of potentially pathogenic Acanthamoeba isolated from the environment in Cairo, Egypt. Parasitol Res 2016; 115(5): 1871-81. [http://dx.doi.org/10.1007/s00436-016-4927-3] [PMID: 26841771]

[342] Dart JKG, Saw VPJ, Kilvington S. Acanthamoeba keratitis: Diagnosis and treatment update 2009. Am J Ophthalmol 2009; 148(4): 487-499.e2.

[http://dx.doi.org/10.1016/j.ajo.2009.06.009] [PMID: 19660733]

[343] Robaei D, Carnt N, Minassian DC, Dart JK. The impact of topical corticosteroid use before diagnosis on the outcome of Acanthamoeba 
keratitis. Ophthalmology 2014; 121(7): 1383-8. [http://dx.doi.org/10.1016/j.ophtha.2014.01.031] [PMID: 24630688]

[344] Carnt N, Robaei D, Watson SL, Minassian DC, Dart JK. Impact of topical corticosteroids used in conjunction with antiamoebic therapy on the outcome of acanthamoeba keratitis. Ophthalmology 2016; 123(5): 984-90.

[http://dx.doi.org/10.1016/j.ophtha.2016.01.020] [PMID: 26952591]

[345] Lorenzo-Morales J, Khan NA, Walochnik J. An update on Acanthamoeba keratitis: Diagnosis, pathogenesis and treatment. Parasite 2015; 22: 10.

[http://dx.doi.org/10.1051/parasite/2015010] [PMID: 25687209]

[346] Martin-Perez T, Criado-Fornelio A, Martinez J, Blanco MA, Fuentes I, Perez-Serrano J. Isolation and molecular characterization of Acanthamoeba from patients with keratitis in Spain. European Journal of Protistology 2017; 61(Pt A): 244-52.

[http://dx.doi.org/10.1016/j.ejop.2017.06.009]

[347] Wynter-Allison Z, Lorenzo Morales J, Calder D, Radlein K, OrtegaRivas A, Lindo JF. Acanthamoeba infection as a cause of severe keratitis in a soft contact lens wearer in Jamaica. Am J Trop Med Hyg 2005; 73(1): 92-4.

[http://dx.doi.org/10.4269/ajtmh.2005.73.92] [PMID: 16014841]

[348] López-Arencibia A, Reyes-Batlle M, Freijo MB, et al. In vitro activity of $1 \mathrm{H}$-phenalen-1-one derivatives against Acanthamoeba castellanii Neff and their mechanisms of cell death. Exp Parasitol 2017; 183: 218-23.

[http://dx.doi.org/10.1016/j.exppara.2017.09.012] [PMID: 28916457]

[349] Hajialilo E, Behnia M, Tarighi F, Niyyati M, Rezaeian M. Isolation and genotyping of Acanthamoeba strains (T4, T9, and T11) from amoebic keratitis patients in Iran. Parasitol Res 2016; 115(8): 3147-51. [http://dx.doi.org/10.1007/s00436-016-5072-8] [PMID: 27102637]

[350] Maghsood AH, Sissons J, Rezaian M, Nolder D, Warhurst D, Khan NA. Acanthamoeba genotype T4 from the UK and Iran and isolation of the T2 genotype from clinical isolates. J Med Microbiol 2005; 54(Pt 8): $755-9$.

[http://dx.doi.org/10.1099/jmm.0.45970-0] [PMID: 16014429]

[351] Xuan YH, Chung BS, Hong YC, Kong HH, Hahn TW, Chung DI. Keratitis by Acanthamoeba triangularis: Report of cases and characterization of isolates. Korean J Parasitol 2008; 46(3): 157-64. [http://dx.doi.org/10.3347/kjp.2008.46.3.157] [PMID: 18830055]

[352] González-Robles A, Omaña-Molina M, Salazar-Villatoro L, et al. Acanthamoeba culbertsoni isolated from a clinical case with intraocular dissemination: Structure and in vitro analysis of the interaction with hamster cornea and MDCK epithelial cell monolayers. Exp Parasitol 2017; 183: 245-53.

[http://dx.doi.org/10.1016/j.exppara.2017.09.018] [PMID: 28974450]

[353] Megha K, Sharma M, Gupta A, Sehgal R, Khurana S. Protein profiling of Acanthamoeba species using MALDI-TOF MS for specific identification of Acanthamoeba genotype. Parasitol Res 2018; 117(3): 729-36.

[http://dx.doi.org/10.1007/s00436-017-5743-0] [PMID: 29344802]

[354] Luo X, Li J, Chen C, Tseng S, Liang L. Ocular demodicosis as a potential cause of ocular surface inflammation. Cornea 2017; 36(Suppl. 1): S9-S14.

[http://dx.doi.org/10.1097/ICO.0000000000001361] [PMID: 28902017]

[355] Lorenzo-Morales J, Martínez-Carretero E, Batista N, et al. Early diagnosis of amoebic keratitis due to a mixed infection with Acanthamoeba and Hartmannella. Parasitol Res 2007; 102(1): 167-9. [http://dx.doi.org/10.1007/s00436-007-0754-x] [PMID: 17899193]
[356] Abedkhojasteh H, Niyyati M, Rahimi F, Heidari M, Farnia S, Rezaeian M. First report of Hartmannella keratitis in a cosmetic soft contact lens wearer in Iran. Iran J Parasitol 2013; 8(3): 481-5. [PMID: 24454444]

[357] Li Z, Breitwieser FP, Lu J, et al. Identifying corneal infections in formalin-fixed specimens using next generation sequencing. Invest Ophthalmol Vis Sci 2018; 59(1): 280-8.

[http://dx.doi.org/10.1167/iovs.17-21617] [PMID: 29340642]

[358] Cali A, Meisler DM, Lowder CY, et al. Corneal microsporidioses: Characterization and identification. J Protozool 1991; 38(6): 215S-7S. [PMID: 1818175]

[359] Joseph J, Sharma S, Murthy SI, et al. Microsporidial keratitis in India: 16S rRNA gene-based PCR assay for diagnosis and species identification of microsporidia in clinical samples. Invest Ophthalmol Vis Sci 2006; 47(10): 4468-73.

[http://dx.doi.org/10.1167/iovs.06-0376] [PMID: 17003441]

[360] Parmar TJ, Gajjar DU, Pal AK, Ghodadra BK. Fungal keratitis associated with mite embedded in cornea. Indian J Pathol Microbiol 2011; 54(1): 214-5

[http://dx.doi.org/10.4103/0377-4929.77415] [PMID: 21393927]

[361] Niyyati M, Lorenzo-Morales J, Rezaie S, et al. First report of a mixed infection due to Acanthamoeba genotype T3 and Vahlkampfia in a cosmetic soft contact lens wearer in Iran. Exp Parasitol 2010; 126(1): 89-90.

[http://dx.doi.org/10.1016/j.exppara.2009.10.009] [PMID: 19857491]

[362] Pinna A, Porcu T, Boscia F, Cano A, Erre G, Mattana A. Free living amoebae [FLA] keratitis. Acta Ophthalmologica Conference. 94.

[363] Ozkoc S, Tuncay S, Delibas SB, et al. Identification of Acanthamoeba genotype T4 and Paravahlkampfia sp. from two clinical samples. J Med Microbiol 2008; 57(Pt 3): 392-6.

[http://dx.doi.org/10.1099/jmm.0.47650-0] [PMID: 18287307]

[364] Chirinos-Saldaña P, Bautista de Lucio VM, Hernandez-Camarena JC, et al. Clinical and microbiological profile of infectious keratitis in children. BMC Ophthalmol 2013; 13: 54

[http://dx.doi.org/10.1186/1471-2415-13-54] [PMID: 24131681]

[365] Gaujoux T, Borsali E, Gavrilov JC, et al. Fungal keratitis caused by Cylindrocarpon lichenicola. J Fr Ophtalmol 2012; 35(5): 356.e1-5. [PMID: 22137679]

[366] Bharathi MJ, Ramakrishnan R, Meenakshi R, Padmavathy S, Shivakumar C, Srinivasan M. Microbial keratitis in South India: influence of risk factors, climate, and geographical variation. Ophthalmic Epidemiol 2007; 14(2): 61-9.

[http://dx.doi.org/10.1080/09286580601001347] [PMID: 17464852]

[367] Chen WL, Wu CY, Hu FR, Wang IJ. Therapeutic penetrating keratoplasty for microbial keratitis in Taiwan from 1987 to 2001. Am J Ophthalmol 2004; 137(4): 736-43.

[PMID: 15059714]

[368] Willcox MD, Holden BA. Contact lens related corneal infections. Biosci Rep 2001; 21(4): 445-61.

[http://dx.doi.org/10.1023/A:1017991709846] [PMID: 11900321]

[369] Clinch TE, Palmon FE, Robinson MJ, Cohen EJ, Barron BA, Laibson PR. Microbial keratitis in children. Am J Ophthalmol 1994; 117(1): 65-71.

[http://dx.doi.org/10.1016/S0002-9394(14)73016-8] [PMID: 8291594]

[370] Munir WM, El Mallah MK, Janda WM, Tu EY. Gemella haemolysans infectious crystalline keratopathy. Cornea 2008; 27(2): 258. [1]. [http://dx.doi.org/10.1097/ICO.0b013e31815b8535] [PMID: 18216595]

\section{(C) 2019 Bartimote et al.}

This is an open access article distributed under the terms of the Creative Commons Attribution 4.0 International Public License (CC-BY 4.0), a copy of which is available at: https://creativecommons.org/licenses/by/4.0/legalcode. This license permits unrestricted use, distribution, and reproduction in any medium, provided the original author and source are credited. 